Publ. RIMS, Kyoto Univ.

Vol. 6 (1970), 257-292

\title{
Infinite Tensor Products of von Neumann Algebras, II
}

By

\author{
Yoshiomi NAKAGAmI*
}

\section{§1. Introduction}

This paper is a continuation of a previous one with the same title [6], in which we have shown the following results on infinite tensor products of operators and those of normal positive linear functionals:

Theorem 3.2. Assume that $\Pi\left\|T_{\imath}\right\| \leqq+\infty$.

I. If $T_{\iota} \in \mathfrak{R}_{\iota}$, then the following three conditions are equivalent:

(i) $\otimes T_{\iota} \in \otimes \mathfrak{N}_{\imath}$.

(ii) For any $\mathfrak{c} \in \Gamma$ and any $\left(x_{\imath}\right) \in \mathfrak{c},\left(T_{\iota} x_{\iota}\right) \in \mathfrak{c}$ or $\otimes T_{\iota} x_{\iota}=0$.

(iii) $\otimes T_{\imath}$ is a strong limit of $\left\{T_{J}: J \Subset I\right\}$, where $T_{J}=\left(\otimes_{J} T_{\iota}\right) \otimes 1\left(J^{c}\right)$ for $J \ll I$.

II. If $T_{\iota} \in \mathfrak{N}_{\iota}^{+}$, then $\otimes T_{\iota} \in\left(\otimes \mathfrak{U}_{\iota}\right)^{+}$.

III. If $T_{\iota} \in \mathfrak{N}_{\imath}^{\prime}$, then $\otimes T_{\iota} \in\left(\otimes \mathfrak{N}_{\iota}\right)^{\prime}$.

Theorem 4.2. Let $\mathfrak{A}_{.}$be a finite von Neumann algebra with the coupling operator $C_{\iota}$ for every $\iota \in I$.

(i) Let $\varphi_{\iota}$ be a normal trace on $\mathfrak{A}_{\iota}$ for each $\iota \in I$ such that $0<\Pi \varphi_{\iota}(1)<+\infty$. If $\sum \varphi_{\iota}\left(\left(1-C_{\imath}\right)^{+}\right)<+\infty$, then there is one and only one normal trace $\varphi$ on $\otimes \mathscr{N}_{\text {. }}$ such that $\varphi\left(\Pi_{K} \bar{A}_{\imath}\right)=\left(\Pi_{K} \varphi_{\iota}\left(A_{\imath}\right)\right)\left(\Pi_{K} c \varphi_{\iota}(1)\right)$ for $A_{\iota} \in \mathfrak{U}_{\iota}$ and every $K \ll I$.

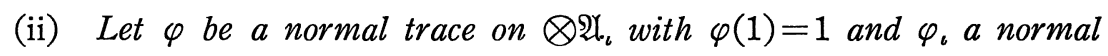
trace corresponding to the restriction of $\varphi$ to $\overline{\mathfrak{Q}}_{2}$ by the natural isomorphism between $\mathfrak{N}_{\iota}$ and $\overline{\mathfrak{A}}_{\iota}$. If $\varphi\left(\Pi_{K} \bar{A}_{\iota}\right)=\Pi_{K} \varphi_{\iota}\left(A_{\iota}\right)$ for $A_{\iota} \in \mathfrak{A}_{\iota}$ and every $K \ll I$,

Received February 9, 1970.

Communicated by $\mathrm{H}$. Araki.

* Department of Mathematics, Tokyo Institute of Technology. 
then $\sum \varphi_{\iota}\left(\left(1-C_{\iota}\right)^{+}\right)<+\infty$.

Corollary 4.2. Let $\mathfrak{A}_{.}$be a von Neumann algebra and $\varphi_{\iota}$ a normal positive linear functional on $\mathfrak{A}_{\iota}$ for each $\iota \in I$. Let $\mathrm{c}$ be a characteristic class of $\left(\varphi_{\iota}\right)$. Let $G_{\iota}$ and $G$ be the carrier projections of $\varphi_{\iota}$ and $\otimes \varphi_{\iota}$ respectively. Then $G=\left(\otimes G_{\iota}\right) P(\mathfrak{c})$.

Making use of these results we will try to give an explicit one to one correspondence between a normalized finite normal trace $\varphi$ on an infinite tensor product of von Neumann algebras and a probability measure $\nu$ on an infinite product of compact spaces. We shall use the reduction theory of von Neumann algebras which has been systematically studied by von Neumann [11], Segal [8] and others. The terminologies and notations concerning the reduction theory will be taken from Dixmier [4].

In section 3 we will consider a correspondence of a normal positive linear functional on an incomplete infinite tensor product of von Neumann algebras to a projective system, with some additional conditions, of normal positive linear functionals of finite tensor products of von Neumann algebras, in analogy with the Kolmogoroff's extension theorem on probability measures. Through the aid of such a consideration on states, we will find that if $\mathfrak{A}_{\text {, }}$ is a finite factor for each $\iota \in I$, then the finite part of $\otimes \mathscr{N}_{\iota}$ is carried by one minimal central projection $P(\mathfrak{c})$ whenever it exists in $\otimes \mathscr{A}_{\iota}$. This corollary enables us to continue a further investigation on the structures of a finite normal trace on $\otimes \mathfrak{N}_{\bullet}$.

In section 4 we will give a partial answer to the problem which we have been aiming at from the beginning of our study of infinite tensor products of von Neumann algebras. The main argument will be presented as a series of lemmas mainly about the decomposition of infinite tensor products of Hilbert spaces, vectors, operators, von Neumann algebras and traces. The final conclusion of the present paper can be summarized as

Theorem. Let $Z_{\iota}$ be a compact Hausdorff space, $\nu_{\iota}$ a probability Radon measure with the carrier $Z_{\iota}, \zeta_{\iota} \rightarrow \mathfrak{C}_{\iota}\left(\zeta_{\iota}\right)$ a $\nu_{\iota}$-measurable field of non zero Hilbert spaces on $Z_{\iota}, \zeta_{\iota} \rightarrow \mathbb{Y}_{\iota}\left(\zeta_{\iota}\right)$ a $\nu_{\iota}$-measurable field of von Neumann algebras on $\mathfrak{E}_{\imath}\left(\zeta_{\iota}\right)$ on $Z_{\iota}$, 


$$
\mathfrak{S}_{\iota}=\int_{Z_{\iota}}^{\oplus} \mathfrak{S}_{\iota}\left(\zeta_{\iota}\right) d \nu_{\iota}\left(\zeta_{\iota}\right), \quad \mathfrak{N}_{\iota}=\int_{Z_{\iota}}^{\oplus} \mathfrak{N}_{\iota}\left(\zeta_{\iota}\right) d \nu_{\iota}\left(\zeta_{\iota}\right)
$$

and $\mathfrak{3}_{\imath}$ the algebra of diagonalizable operators on $\mathfrak{S}_{2}$. Assume that $I$ is countable, $Z_{\iota}$ satisfies the second axiom of countability, $\mathfrak{H}_{\iota}$ is finite, $\mathfrak{Z}_{\iota}$ is the center of $\mathfrak{X}_{.}$and $\sum\left\|\left(1-C_{\imath}\right)^{+}\right\|<+\infty$ where $C_{\iota}$ is the coupling operator of $\mathfrak{A}_{.}$. Then there is one and only one finite $P\left(\mathfrak{c}_{0}\right), \mathfrak{c}_{0} \in \Gamma$, in $\left\{P\left(\mathfrak{c}^{\prime}\right): \mathfrak{c}^{\prime} \in \Gamma\right\}$ and there is a $\otimes \nu_{\iota}$-measurable field $\zeta \rightarrow \psi_{\zeta}^{c}$ of normal traces on $Z=\Pi Z$ 。 for each $P_{\mathfrak{c}} \leqq P\left(\mathfrak{c}_{0}\right)$ with the following two properties:

(i) If $\varphi$ is a normal trace on $\otimes \Re_{\text {. }}$ with $\varphi(1)=1$, then there is a probability measure $\nu$ on $Z$, absolutely continuous with respect to $\otimes \nu_{\iota}$, such that the restriction of $\varphi$ to $\otimes^{\mathrm{c}} \mathfrak{Q}_{2}$ is

$$
\int_{Z}^{\oplus} \psi_{\zeta}^{\mathrm{c}} d \nu(\zeta)
$$

(ii) if $\nu$ is a probability measure on $Z$, absolutely continuous with respect to $\otimes \nu_{\iota}$, then

$$
\varphi=\int_{Z}^{\oplus} \psi_{\zeta}^{c} d \nu(\zeta)
$$

exists and is a normal trace on $\otimes \mathfrak{N}^{-}$with $\varphi(1)=1$, whose extension to $\otimes \mathfrak{N}_{.}$is also a finite normal trace on $\otimes \mathfrak{H}_{\llcorner}$.

\section{§2. Preparatory Notations and Definitions}

In what follows we will have to assume that the reader is familiar with the elementary properties of von Neumann algebras which are given in $[4]$.

von Neumann algebra: Let $\mathfrak{S}$ be a Hilbert space, $x$ a vector in $\mathfrak{S}$ and $\mathfrak{H}$ a von Neumann algebra on $\mathfrak{S}$. $\boldsymbol{C}_{\mathfrak{g}}$ and $\mathfrak{L}(\mathfrak{E})$ stand for von Neumann algebras of all scalar operators and all operators on $\mathfrak{S}$ respectively. Denote by $E(\mathfrak{R}, x)$ the projection onto the subspace spanned by $\{A x: A \in \mathfrak{R}\}$. Let 0 and 1 denote the zero and the identity operators. By $\mathfrak{A}^{u}, \mathfrak{A}^{+}$, and $\mathfrak{2} .^{p}$ we mean the set of all unitary, non negative and projection operators in $\mathfrak{2}$ respectively. $\quad \omega_{x}$ is a positive linear functional defined by $\omega_{x}(A)=(A x, x)$ for $A \in \mathfrak{A}$. A normal trace $\psi$ on a factor is called the canonical trace if 
$\phi(1)=1$. Let $\varphi$ be a normal positive linear functional on 2 .. Define the characteristic number $\gamma$ of $\varphi$ by $\gamma=\sup \left\{\|x\|^{2}: \varphi-\omega_{x} \geqq 0\right\}$. If $\gamma=\omega_{x}(1)$, then $x$ is called a characteristic vector of $\varphi$. Let $\rho$ be a semi-finite faithful normal trace of a von Neumann algebra $\mathfrak{N}$ on a Hilbert space $\mathfrak{g}$. Then we have a gage space $(\mathfrak{C}, \mathfrak{R}$., $\rho)$ in the sence of Segal [9]. Denote by $L^{1}(\mathfrak{A}$, $\rho)$ or $L^{1}(\rho)$ the set of all integrable operators and by $L^{2}(\mathscr{A}$., $\rho)$ or $L^{2}(\rho)$ the set of all square integrable operators. Such a concept can be generalized to the case where $\rho$ is not necessarily faithful by restricting the argument to the carrier space of $\rho$.

Infinite tensor product of Hilbert spaces: Let $I$ be an infinite index set. We shall denote $J \ll I$ whenever $J$ is a finite subset of $I$. We shall use the symbols $\Sigma, \Pi, \cup$ and $\cap$ without index set $I . z^{+}$is the set of non negative integers. Let $\mathfrak{S}_{\iota}$ be a non trivial Hilbert space, $e_{\iota}$, $x_{\imath}, \ldots$ the vectors of $\mathfrak{S}_{\imath}$ and \|\| the norm on $\mathfrak{S}_{\imath}$. If $0<\Pi\left\|x_{\iota}\right\|<+\infty$ for $x_{\iota} \in \mathfrak{S}_{\iota}$, then the set $\left\{x_{\iota}: \iota \in I\right\}$ is called a $C_{0}$-sequence and written by $\left(x_{\imath}\right)$. A pair of $\left(x_{\iota}\right)$ and $\left(y_{\imath}\right)$ is equivalent if $\Sigma\left|\left(x_{\iota}, y_{\imath}\right)-1\right|<+\infty$, which we denote by $\left(x_{\imath}\right) \sim\left(y_{\imath}\right)$. Denote the set of all $C_{0}$-sequences by $\Gamma_{0}$ and the set of equivalence classes $c$ by $\Gamma=\Gamma_{0} / \sim$. Let $\otimes \mathfrak{S}_{2}$ denote the complete infinite tensor product of $\mathfrak{S}_{\imath}$ and $\odot \mathfrak{E}_{\imath}$ the set of all finite linear combinations of tensor product vectors $\otimes x_{\imath}$ for $\left(x_{\imath}\right) \in \Gamma_{0}$. Let $\otimes^{\prime} \mathfrak{S}_{\imath}$ denote the incomplete infinite tensor product of $\mathfrak{E}_{\text {。 }}$ with respect to $c \in \Gamma$.

Infinite tensor product of von Neumann algebras: Let $\mathfrak{H}_{c}$ be von Neumann algebras on $\mathfrak{S}_{\iota}$ for each $\iota \in I$. Denote the zero operator and the identity operator on $\mathfrak{S}_{\iota}$ by $0_{\iota}$ and $1_{\iota}$, sometimes without suffix. $1(J)$ is the identity on $\otimes_{J} \mathfrak{S}_{\iota}$. Denote $\bar{A}_{\iota}=A_{\iota} \otimes 1(I-\{c\})$. $\otimes \mathscr{U}_{\iota}$ is a von Neumann algebra on $\otimes \mathfrak{S}_{\iota}$ generated by $\bar{A}_{\iota}$ satisfying $A_{\iota} \in \mathfrak{N}_{\iota}$ for all $\iota \in I$. Let $\odot \mathscr{R}_{\iota}$ be the union of $\Pi_{J}\left\{\bar{A}_{\iota}: A_{\iota} \in \mathfrak{N}_{\iota}\right\}$ for all $J \ll I . \quad P_{\mathrm{c}}$ is a projection of $\otimes \mathfrak{S}_{\text {, to }} \otimes^{\mathfrak{c}} \mathfrak{S}_{\text {, for }} \mathfrak{c} \in \Gamma$ and $P(\mathfrak{c})$ is its central carrier in $\left(\otimes \mathscr{N}_{\imath}\right)^{\prime}$. Since $P(\mathfrak{c})=\lim 1(J) \otimes E\left(\left(\otimes_{J} c \mathfrak{N}_{\imath}\right)^{\prime}, \otimes_{J^{c}} x_{\iota}\right)$ for $\left(x_{\iota}\right) \in \mathfrak{c}, P(\mathfrak{c})$ belongs to the commutor of $\odot \mathbb{Q}\left(\mathfrak{E}_{\imath}\right)$ and hence $P(\mathfrak{c}) \in\left(\otimes \mathbb{Q}\left(\mathfrak{E}_{\imath}\right)\right)^{\prime}$. Since $P(\mathfrak{c}) \in \otimes \mathscr{N}_{\cdot}$ and $\otimes \mathscr{N}_{.} \subset \otimes \mathbb{N}\left(\mathfrak{E}_{\imath}\right)$, it follows that $P(\mathfrak{c})$ is a central projection of

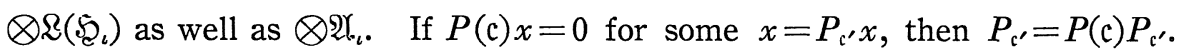
Consequently, $P(\mathfrak{c})=\sum_{\mathfrak{c}^{\prime} \in \Gamma, P_{\mathfrak{c}} \leqq P(\mathrm{c})} P_{\mathrm{c}^{\prime}} . \quad \otimes^{\mathfrak{c}} A_{\iota}$ or $\otimes^{(\mathrm{c})} A_{\iota}$ is the restriction of 
$P_{\mathrm{c}}\left(\otimes A_{\iota}\right) P_{\mathrm{c}}$ or $P(\mathrm{c})\left(\otimes A_{\iota}\right)$ to $P_{\mathrm{c}}$ or $P(\mathrm{c})$ respectively. The set of operators $A_{E}$ which are the restriction of $E A E$ to $E$ for $A \in \otimes \mathscr{R}_{\text {c }}$ where $E=P_{\mathrm{c}}$ or $P(\mathrm{c})$, forms a von Neumann algebra, which is denoted by $\otimes^{(\mathfrak{N}} \mathfrak{H}_{\iota}$ or $\otimes^{(c)} \mathfrak{Q}_{l}$. Similarly $\odot \mathfrak{C S}_{\text {, }}$ or $\odot{ }^{(\mathrm{c})} \mathfrak{Q}_{\text {, }}$ is also used. Let $\varphi$ be a positive linear functional on $\otimes \mathscr{R}_{.}$. For any $A \in \otimes_{J} \mathfrak{K}_{\text {. }}$ we define $\psi(A)=\varphi\left(A \otimes 1\left(J^{C}\right)\right)$, then $\psi$ is a positive linear functional on $\otimes_{J} \mathfrak{2}_{l}$, which is called the projection of $\varphi$ to $\otimes_{J} \mathfrak{A}_{\iota}$. Let $\varphi_{\iota}$ be a positive linear functional on $\mathfrak{N}_{.}$with a characteristic vector $x_{\iota}$. The equivalence class $\mathrm{c} \in \Gamma$ which contains $\left(x_{\iota}\right)$ is called a characteristic class of $\left(\varphi_{\iota}\right)$.

Let $J \ll I$. Then the similar arguments about infinite tensor products of Hilbert spaces and of von Neumann algebras hold for $I-J$ instead of I. As it is easily seen that there is a canonical one to one correspondence between the equivalence classes of $C_{0}$-sequences with respect to $I$ and $I-J$, we shall use the same notation $c$ for the equivalence classes unless any confusions arise.

The measure which appears in this paper is a Radon measure. It is however known that the concept of measure, Radon or Borel coincide on the completely regular space if it is the surjective image of a Polish space by a continuous mapping $[7]$.

Finally recall some results on the decomposition of von Neumann algebra, which are referred to $[4]$. Let $\zeta \rightarrow \mathfrak{S}(\zeta)$ be a field of Hilbert spaces on a locally compact Hausdorff space $Z$ and $\nu$ a positive Radon measure on $Z$ whose carrier is $Z$. We say that $\mathfrak{S}(\zeta)$ forms a $\nu$-measurable field of Hilbert spaces if a vector subspace $\$ S$ of $\prod_{\zeta \in Z} \mathfrak{(}(\zeta)$ with the following properties is given:

(i) For every $x \in \mathbb{S}$ the function $\zeta \rightarrow\|x(\zeta)\|$ is $\nu$-measurable;

(ii) If $y \in \Pi_{\zeta \in Z} \mathfrak{E}(\zeta)$ is such that, for every $x \in \mathbb{B}$, the function $\zeta \rightarrow(x(\zeta), y(\zeta))$ is $\nu$-measurable, then $y \in \mathbb{B S}$;

(iii) There exists a sequence $\left\{x_{1}, x_{2}, \ldots\right\}$ of elements of (B) such that, for $\zeta \in Z,\left\{x_{n}(\zeta): n=1,2, \ldots\right\}$ forms a total sequence in $\mathscr{S}(\zeta)$.

The property (iii) implies that $\mathfrak{S}(\zeta)$ is separable. If the followings are given:

$1^{\circ}$ a $\nu$-measurable field $\zeta \rightarrow \mathfrak{C}(\zeta)$ of non zero Hilbert spaces on $Z$; 
$2^{\circ}$ a $\nu$-measurable field $\zeta \rightarrow \mathfrak{N}$. $(\zeta)$ of von Neumann algebras over $\mathfrak{C}(\zeta)$ on $Z$, then we can construct canonically:

$1^{\circ}$ the Hilbert space

$$
\mathfrak{Q}=\int_{Z}^{\oplus} \mathfrak{S}(\zeta) d \nu(\zeta)
$$

$2^{\circ}$ the von Neumann algebra 3 of diagonalizable operators which is isomorphic to $L^{\infty}(Z, \nu)$;

$3^{\circ}$ the decomposable von Neumann algebra

$$
\mathfrak{A}=\int_{Z}^{\oplus} \mathfrak{A}(\zeta) d \nu(\zeta) .
$$

In this case we call $\mathfrak{g}(\zeta), \mathfrak{N}(\zeta), \ldots$ a stalk of $\mathfrak{S}, \mathfrak{A}$, $\ldots$ at $\zeta \in Z$ respectively. In section 4 we shall employ the notation indexed by $\iota \in I$,

$$
Z_{\iota}, \nu_{\iota}, \zeta_{\iota}, \mathfrak{S}_{\iota}\left(\zeta_{\iota}\right), \mathfrak{N}_{\iota}\left(\zeta_{\iota}\right), \mathfrak{S}_{\iota}, \mathfrak{B}_{\iota} \text { and } \mathfrak{N}_{\iota}
$$

instead of the corresponding ones in the above respectively.

\section{§3. Normal Positive Linear Functional on $\otimes^{\mathfrak{c}} \mathrm{A}_{\text {. }}$}

Since $P(c)$ for $c \in \Gamma$ is the central carrier of $P_{\mathrm{c}}$, the study of a normal positive linear functional on $\otimes \mathscr{N}_{.}$, is reduced to that on each $\otimes^{(c)} \mathfrak{U}_{\iota}$ and

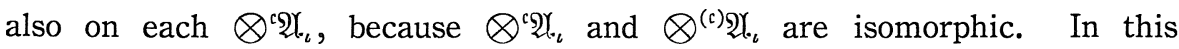
section we shall give a characterization of a normal positive linear functional on an incomplete infinite tensor product $\otimes^{\mathfrak{r}} \mathfrak{Y}_{6}$.

Throughout this section we shall assume that $\left(e_{\iota}\right) \in \mathrm{c}$ and $\left\|e_{\iota}\right\|=1$, denote $e(K)=\bigotimes_{K} e_{\iota}$ for every $K \subset I$, and use the same notation $\omega_{e(K)}$ for $\omega_{e(K)}(\cdot)=(\cdot e(K), e(K))$ on $\bigotimes_{K} \mathfrak{H}_{\iota}, \bigotimes_{K}^{c} \mathfrak{A}_{\iota}$ and $\bigotimes_{K}^{(c)} \mathfrak{A}_{\iota}$.

Lemma 3.1. Let $\otimes^{\mathfrak{N}}$. be an incomplete infinite tensor product of $\mathfrak{H}_{\imath}$. If $\varphi$ is a normal positive linear functional on $\otimes \mathfrak{N}_{.}$, then for any $\varepsilon>0$ there exists $J_{0} \ll I$ such that

$$
\left\|\varphi-\varphi(J) \otimes \omega_{e\left(J^{c}\right)}\right\|<\varepsilon
$$

for any $J \ll I$ with $J_{0} \subset J$, where $\varphi(J)$ is the projection of $\varphi$ onto $\otimes_{J} \mathfrak{A}_{.}$.

Proof. Since Lemma clearly holds if $\varphi=0$, it suffices to consider the 
case where $\varphi \neq 0$. Since $\varphi$ is a normal positive linear functional, we have

$$
\varphi=\sum_{i=1}^{\infty} \omega_{x_{\imath}} \quad \text { for } \quad x_{i} \in \otimes^{c} \mathfrak{Q}_{\imath}
$$

and $0<\sum_{i=1}^{\infty}\left\|x_{i}\right\|^{2}=\sum_{i=1}^{\infty} \omega_{x_{\imath}}(1)=\varphi(1)<+\infty$. For any $\varepsilon>0$ there exists a positive integer $n, J_{0} \ll I$ and suitable vectors $x_{i}\left(J_{0}\right) \in \otimes_{J_{0}} \mathfrak{E}_{\iota}, i=1,2, \ldots, n$ such that $\sum_{i=n+1}^{\infty}\left\|x_{i}\right\|^{2}<\varepsilon / 4$,

$$
\left\|x_{i}-x_{i}\left(J_{0}\right) \otimes e\left(J_{0}^{c}\right)\right\|<\varepsilon \varphi(1)^{-1 / 2} / 8 n \text { and }\left\|x_{i}\left(J_{0}\right) \otimes e\left(J_{0}^{c}\right)\right\| \leqq \varphi(1)^{1 / 2}
$$

for $i=1,2, \ldots, n$. It follows that

$$
\left\|\omega_{x_{\imath}}-\omega_{x_{2}\left(J_{0}\right) \otimes e\left(J_{0}\right)}\right\|<\frac{\varepsilon}{4 n}
$$

for $i=1,2, \ldots, n$, and hence

$$
\begin{aligned}
& \left\|\varphi-\sum_{i=1}^{n} \omega_{x_{\imath}\left(J_{0}\right) \otimes \omega\left(J_{0}^{c}\right)}\right\| \\
& \leqq\left\|\sum_{i=1}^{n}\left(\omega_{x_{\imath}}-\omega_{x_{\imath}\left(J_{0}\right) \otimes e\left(J_{0}^{c}\right)}\right)\right\|+\left\|\sum_{i=n+1}^{\infty} \omega_{x_{\imath}}\right\|<\frac{\varepsilon}{2} .
\end{aligned}
$$

Define $\psi=\sum_{i=1}^{n} \omega_{x_{\imath}\left(J_{0}\right) \otimes e\left(J_{0}^{c}\right)}$. Let $\varphi(J)$ and $\phi(J)$ be the projections of $\varphi$ and $\psi$ onto $\otimes_{J} \mathfrak{H}_{\text {. }}$ respectively. Then we get $\phi(J)=\sum_{i=1}^{n} \omega_{x_{2}(J) \otimes e\left(J-J_{0}\right)}$ and

$$
\|\varphi(J)-\psi(J)\| \leqq\|\varphi-\psi\|<\frac{\varepsilon}{2}
$$

Thus we have

$$
\begin{aligned}
& \left\|\varphi-\varphi(J) \otimes \omega_{e\left(J^{c}\right)}\right\| \\
& \leqq\left\|\varphi-\psi(J) \otimes \omega_{e\left(J^{c}\right)}\right\|+\left\|(\psi(J)-\varphi(J)) \otimes \omega_{e\left(J^{c}\right)}\right\|<\varepsilon . \quad \text { Q.E.D. }
\end{aligned}
$$

Corollary 3.1. Under the same assumption as that of Lemma 3.1, the following conditions (i) and (ii) are equivalent:

(i) $\varphi$ is a normal positive linear functional on $\otimes \mathfrak{2}_{.}$; and

(ii) for any $\varepsilon>0$ there exists $J_{0} \ll I$ such that

$$
\left\|\varphi-\varphi(J) \otimes \omega_{e\left(J^{c}\right)}\right\|<\varepsilon
$$

for any $J \ll I$ with $J_{0} \subset J$, where $\varphi(J)$ is a normal positive linear functional on $\otimes_{J} \mathfrak{H}_{L}$. 
Proof. (ii) implies (i) is obvious. (i) implies (ii) is clear from the proof of the last lemma.

Definition 3.1. The family $\{\varphi(J): J \Subset I\}$ of normal positive linear functionals $\varphi(J)$ on $\otimes_{J} \mathfrak{A}_{.}$for any non empty $J \ll I$ is called a projective system, if $J_{1} \subset J_{2} \ll I$, then the projection of $\varphi\left(J_{2}\right)$ to $\otimes_{J_{1}} \mathfrak{A}_{\iota}$ is $\varphi\left(J_{1}\right)$.

Definition 3.2. A projective system $\{\varphi(J): J \Subset I\}$ is c-normal, if for any $\varepsilon>0$ there exists $J \ll I$ such that

$$
\left\|\varphi(K)-\omega_{e(K)}\right\|<\varepsilon
$$

for every $K \ll J^{c}$.

Definition 3.3. Given a finite family of von Neumann algebras $\mathfrak{2}_{i}$ on $\mathfrak{S}_{i}, i=1,2, \ldots, n$. Let $\varphi$ be a normal positive linear functional on $\bigotimes_{i=1}^{n} \mathfrak{I}_{i}$ and $\varphi_{i}$ its projection to $\mathfrak{H}_{i}$. Define

$$
C\left(\varphi ; \mathfrak{N}_{1}, \ldots, \mathfrak{R}_{n}\right)=\left\|\varphi-\varphi_{1} \otimes \cdots \otimes \varphi_{n}\right\| .
$$

Let $\varphi$ and $\phi$ be normal positive linear functionals on a von Neumann algebra 2r.. We shall say that $\psi$ is absolutely continuous with respect to $\varphi$, if $\varphi(N)=0$ implies $\psi(N)=0$ for $N \in \mathfrak{U}^{p}$.

Lemma 3.2. Let $\varphi$ be a normal positive linear functional on $\bigotimes_{i=1}^{n} \mathfrak{A}_{i}$ and $\varphi_{i}$ its projection to $\mathfrak{\Re}_{i}$. Then $\varphi$ is absolutely continuous with respect to $\bigotimes_{i=1}^{n} \varphi_{i}$.

Proof. As $\varphi$ is normal, we have a countable subset $\mathfrak{M}$ of $\otimes_{i=1}^{n} \mathfrak{S}_{i}$ such that $\varphi=\sum_{x_{\imath} \in \mathfrak{m} \omega_{x_{i}}}$. Therefore the carrier $E$ (resp. $\bar{E}_{j}$ ) of $\varphi$

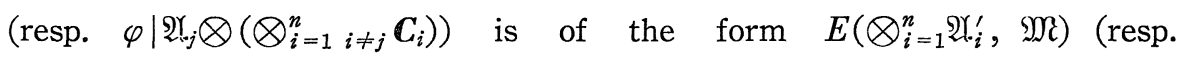
$\left.E\left(\mathfrak{N}_{j}^{\prime} \otimes\left(\otimes_{i=1}^{n}{ }_{i \neq j} \mathbb{R}\left(\mathfrak{S}_{i}\right)\right), \mathfrak{M}\right)\right)$. Utilizing the carrier $E_{j}$ of $\varphi_{j}$, we have $\bar{E}_{j}=E_{j} \otimes\left(\bigotimes_{i=1}^{n} i \neq j 1_{i}\right)$. Since it is clear that $E \leqq \bar{E}_{j}$ for $j=1,2, \ldots, n$, we know

$$
E \leqq \prod_{j=1}^{n} \bar{E}_{j}=E_{1} \otimes \cdots \otimes E_{n}
$$

Since $\bigotimes_{i=1}^{n} E_{i}$ is the carrier of $\bigotimes_{i=1}^{n} \varphi_{i}$, the null projection $N$ of $\bigotimes_{i=1}^{n} \varphi_{i}$ is orthogonal to $\otimes_{i=1}^{n} E_{i}$ and therefore it is orthogonal to $E$. Hence $N$ is also a null projection of $\varphi$.

Q.E.D. 
Remark 3.1. Given finite normal traces $\varphi_{i}$ on $\mathfrak{A}_{i}(i=1,2, \ldots, n)$ and a normal positive linear functional $\varphi$ on $\otimes_{i=1}^{n} \mathfrak{A}_{i}$, then there exists a non negative operator $D$ in $L^{1}\left(\bigotimes_{i=1}^{n} \mathfrak{H}_{i}, \bigotimes_{i=1}^{n} \varphi_{i}\right)$ such that

$$
\varphi(A)=\left(\varphi_{1} \otimes \cdots \otimes \varphi_{n}\right)(D A)
$$

for $A \in \bigotimes_{i=1}^{n} \mathfrak{A l}_{i}$ by Dye [5]. Such a $D$ is called the Radon-Nikodym derivative of $\varphi$ with respect to $\otimes_{i=1}^{n} \varphi_{i}$. Let $D=\int \lambda d E_{\lambda}$ be the spectral resolution of $D$ and define

$$
G=\int_{0}^{1} d E_{\lambda}
$$

Then we have

$$
\begin{aligned}
\| \varphi-\varphi_{1} \otimes \cdots \otimes \varphi_{n} \mid i= & \left(\varphi_{1} \otimes \cdots \otimes \varphi_{n}\right)((1-D) G) \\
& +\left(\varphi_{1} \otimes \cdots \otimes \varphi_{n}\right)((D-1)(1-G)) \\
= & \left(\varphi_{1} \otimes \cdots \otimes \varphi_{n}\right)(|1-D|) .
\end{aligned}
$$

Thus, in particular, if $\varphi$ is a finite normal trace and $\varphi_{i}$ is its projection to $\mathfrak{Q}_{i}, i=1,2, \ldots, n$, then

$$
C\left(\varphi ; \mathfrak{N}_{1}, \ldots, \mathfrak{N}_{n}\right)=\left(\varphi_{1} \otimes \cdots \otimes \varphi_{n}\right)(|1-D|),
$$

where $D$ is the Radon-Nikodym derivative of $\varphi$ with respect to $\bigotimes_{i=1}^{n} \varphi_{i}$.

Remark 3.2. Let $\mathfrak{A}_{-i}$ be a finite von Neumann algebra and $\varphi_{i}$ a faithful normal trace on $\mathfrak{A}_{i}$ with $\varphi_{i}(1)=1$ for $i=1,2, \ldots, n$. Then $\varphi=\bigotimes_{i=1}^{n} \varphi_{i}$ is a faithful normal trace on $\bigotimes_{i=1}^{n} \mathfrak{A}_{i}$ and $L^{2}(\varphi)=\bigotimes_{i=1}^{n} L^{2}\left(\varphi_{i}\right)$. Let $\psi_{i}$ and $\psi$ be normal positive linear functionals on $\mathfrak{U}_{i}$ and $\otimes_{i=1}^{n} \mathfrak{A}_{i}$ such that $\psi_{i}(1)=\psi(1)=1$ and $\psi$ is absolutely continuous with respect to $\otimes_{i=1}^{n} \psi_{i}$. Then there are Radon-Nikodym derivatives $D=d \psi / d \varphi$ in $L^{1}(\varphi), D_{i}=d \psi_{i} / d \varphi_{i}$ in $L^{1}\left(\varphi_{i}\right)$ and $T$ in $L^{2}\left(\otimes_{i=1}^{n} \psi_{i}\right)$ with $\phi(\cdot)=\left(\otimes_{i=1}^{n} \psi_{i}\right)\left(T \cdot T^{*}\right)$. Since

$$
\psi(\cdot)=\left(\bigotimes_{i=1}^{n} \psi_{i}\right)\left(T \cdot T^{*}\right)=\varphi\left(T^{*}\left(\bigotimes_{i=1}^{n} D_{i}\right) T \cdot\right),
$$

we have $D=T^{*}\left(\otimes_{i=1}^{n} D_{i}\right) T$ in $L^{1}(\varphi)$. In particular, if each $\psi_{i}$ is a trace for $i=1,2, \ldots, n$, then $D=\left(\otimes_{i=1}^{n} D_{i}\right)|T|^{2}$, and hence, since $D^{1 / 2}$ and $\bigotimes_{i=1}^{n} D_{i}^{1 / 2}$ are vectors in a Hilbert space $L^{2}(\varphi)$, we may consider their 
inner product with respect to $\varphi$

$$
\left(D^{1 / 2}, \bigotimes_{i=1}^{n} D_{i}^{1 / 2}\right)=\varphi\left(\left(\bigotimes_{i=1}^{n} D_{i}^{1 / 2}\right) D^{1 / 2}\right)=\left(\bigotimes_{i=1}^{n} \psi_{i}\right)(|T|),
$$

which is a generalization of the quantity introduced by Araki $[1 ;$ p. 1360] and Araki and Woods [2; p. 166], but which is somewhat different from ours;

$$
\left\|\psi-\bigotimes_{i=1}^{n} \psi_{i}\right\|=\left(\bigotimes_{i=1}^{n} \psi_{i}\right)\left(\left.|1-| T\right|^{2} \mid\right)
$$

by Remark 3.1, since $|T|^{2}$ is the Radon-Nikodym derivative of $\psi$ with respect to $\bigotimes_{i=1}^{n} \psi_{i}$.

Theorem 3.1. Let $\otimes \mathfrak{A N}_{.}$be an incomplete infinite tensor product of

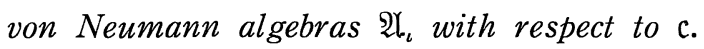

(i) If $\varphi$ is a normal normalized positive linear functional on $\otimes^{\mathfrak{A}}$. $_{\text {, }}$, then the family $\{\varphi(J): J \Subset I\}$ of projections $\varphi(J)$ of $\varphi$ to $\otimes_{J} \mathfrak{A}_{\text {. }}$ for $J \ll I$ is a c-normal projective system with

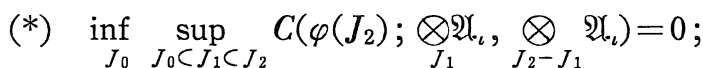

(ii) if $\{\varphi(J): J \Subset I\}$ is a c-normal projective system of normal positive linear functionals $\varphi(J)$ on $\otimes_{J} \mathfrak{N}_{\text {. }}$ with $\left(^{*}\right)$, then there exists uniquely a normalized normal positive linear functional $\varphi$ on $\otimes \mathfrak{A S}_{\text {. }}$ such that $\varphi(J)$ is the projection of $\varphi$ to $\otimes_{J} \mathfrak{A}_{2}$ for each $J \ll I$.

Proof. (i) Let $\varphi(J)$ be the projection of $\varphi$ to $\otimes_{J} \mathfrak{U}_{\iota}$. Then it is clear that $\{\varphi(J): J \Subset I\}$ is a projective system of normal positive linear functionals $\varphi(J)$. According to Lemma 3.1, since $\varphi$ is normal, for any $\varepsilon>0$ there exists $J_{0} \ll I$ such that

$$
\left\|\varphi-\varphi(J) \otimes \omega_{e\left(J^{c}\right)}\right\|<\varepsilon
$$

for any $J \ll I$ with $J_{0} \subset J$. Hence for any $K \ll J_{0}^{c}$ we have

$$
\begin{aligned}
& \left\|\varphi(K)-\omega_{e(K)}\right\| \\
& \quad=\sup _{A \in \otimes_{K} \mathscr{A}_{c},\|A\|=1}\left|\left(\varphi-\varphi\left(J_{0}\right) \otimes \omega_{e\left(J_{0}^{c}\right)}\right)\left(1\left(K^{c}\right) \otimes A\right)\right|<\varepsilon,
\end{aligned}
$$

from which we know that $\{\varphi(J): J \Subset I\}$ is c-normal. Moreover for any $J_{1}$ and $J_{2}$ with $J_{0} \subset J_{1} \subset J_{2} \ll I$, we obtain 


$$
\begin{aligned}
& \left\|\varphi\left(J_{2}\right)-\varphi\left(J_{1}\right) \otimes \varphi\left(J_{2}-J_{1}\right)\right\| \\
& \qquad\left\|\varphi\left(J_{2}\right)-\varphi\left(J_{1}\right) \otimes \omega_{e\left(J_{2}-J_{1}\right)}\right\| \\
& \quad+\left\|\varphi\left(J_{1}\right) \otimes\left(\varphi\left(J_{2}-J_{1}\right)-\omega_{e\left(J_{2}-J_{1}\right)}\right)\right\|<2 \varepsilon .
\end{aligned}
$$

Thus $\inf _{J_{0}} \sup _{J_{0} \subset J_{1} \subset J_{2}} C\left(\varphi\left(J_{2}\right) ; \otimes_{J_{1}} \mathfrak{A}_{\iota}, \otimes_{J_{2}-J_{1}} \mathfrak{A}_{\iota}\right)=0$.

(ii) Let $\{\varphi(J): J \Subset I\}$ be a c-normal projective system. Denote a positive linear functional $\varphi(J) \otimes \omega_{e\left(J^{c}\right)}$ by $\varphi_{J}$, where $\left(e_{\iota}\right) \in c$ and $\left\|e_{\iota}\right\|=1$. From our assumption

$$
\inf _{J_{0}} \sup _{J_{0} \subset J_{1} \subset J_{2}} C\left(\varphi\left(J_{2}\right) ; \underset{J_{1}}{\bigotimes \mathfrak{N}_{. \iota},} \underset{J_{2}-J_{1}}{\bigotimes} \mathfrak{\Re}_{\imath}\right)=0,
$$

it follows that for any $\varepsilon>0$ there exists $J_{3} \ll I$ such that

$$
C\left(\varphi\left(J_{5}\right) ; \underset{J_{4}}{\otimes \mathfrak{U}_{\iota},} \underset{J_{5}-J_{4}}{\otimes} \mathfrak{N}_{\iota}\right)=\left\|\varphi\left(J_{5}\right)-\varphi\left(J_{4}\right) \otimes \varphi\left(J_{5}-J_{4}\right)\right\|<\varepsilon
$$

for any $J_{3} \subset J_{4} \subset J_{5} \ll I$. Since $\{\varphi(J): J \Subset I\}$ is c-normal, for any $\varepsilon>0$ there exists $J_{6} \ll I$ such that

$$
\left\|\varphi\left(J_{7}\right)-\omega_{e\left(J_{7}\right)}\right\|<\varepsilon
$$

for any $J_{7} \ll I$ with $J_{6} \cap J_{7}=\phi$. Denote $K_{0}=J_{3} \cup J_{6}$. Then for any $K_{i} \ll I$ with $K_{0} \subset K_{i}(i=1,2)$

$$
\begin{aligned}
\left\|\varphi_{K_{1}}-\varphi_{K_{2}}\right\| \leqq & \left\|\varphi_{K_{1}}-\varphi_{K_{1} \cap K_{2}}\right\|+\left\|\varphi_{K_{2}}-\varphi_{K_{1} \cap K_{2}}\right\| \\
= & \left\|\varphi\left(K_{1}\right)-\varphi\left(K_{1} \cap K_{2}\right) \otimes \omega_{e\left(K_{1}-K_{2}\right)}\right\| \\
& +\left\|\varphi\left(K_{2}\right)-\varphi\left(K_{1} \cap K_{2}\right) \otimes \omega_{e\left(K_{2}-K_{1}\right)}\right\| \\
\leqq & \left\|\varphi\left(K_{1}\right)-\varphi\left(K_{1} \cap K_{2}\right) \otimes \varphi\left(K_{1}-K_{2}\right)\right\| \\
& +\left\|\varphi\left(K_{1} \cap K_{2}\right) \otimes\left(\varphi\left(K_{1}-K_{2}\right)-\omega_{e\left(K_{1}-K_{2}\right)}\right)\right\| \\
& +\left\|\varphi\left(K_{2}\right)-\varphi\left(K_{1} \cap K_{2}\right) \otimes \varphi\left(K_{2}-K_{1}\right)\right\| \\
& +\left\|\varphi\left(K_{1} \cap K_{2}\right) \otimes\left(\varphi\left(K_{2}-K_{1}\right)-\omega_{e\left(K_{2}-K_{1}\right)}\right)\right\|<4 \varepsilon .
\end{aligned}
$$

Thus $\left\{\varphi_{J}: J \Subset I\right\}$ is a Cauchy net in the uniform topology. So the uniform limit $\varphi=\lim \varphi_{J}$ is a normal positive linear functional satisfying that the projection of $\varphi$ to $\otimes_{J} \mathfrak{A}_{\imath}$ is $\varphi(J)$. Uniqueness is clear from the fact that $\odot \mathfrak{A}^{2}$ is weakly dense in $\otimes \mathfrak{A N}_{\iota}$.

Q.E.D. 
Remark 3.3. In the last theorem the c-normality of $\{\varphi(J): J \Subset I\}$ does not implies the condition $\left(^{*}\right)$ as shown in the following example which is suggested by Kubo. The similar ideas are orally communicated by Araki from physical points.

Let $I=z^{+}, I_{0}=z^{+}-\{0\}, \mathfrak{S}_{\text {}}$ be a two dimensional Hilbert space and $\mathfrak{U}_{\imath}$ be the maximal abelian von Neumann subalgebra of $\mathfrak{L}\left(\mathfrak{\bigvee}_{\iota}\right)$ for $\iota \in I_{0}$. Then $\mathfrak{U}_{\imath}$ is identified with the diagonal matrix algebra, by choosing a suitable complete orthonormal base $\left\{e_{\iota 1}, e_{\iota 2}\right\}$ in $\mathfrak{E}_{\iota}$. Denote the projection of $\mathfrak{Q}_{\imath}$ to the subspace spanned by $e_{\iota j}$ by $E_{\iota j}$ for $j=1$ or 2 . Then $\mathfrak{H}_{\iota}$ is generated by $\left\{E_{\iota 1}, E_{\iota 2}\right\}$. Define $e_{\iota}=(1 / \sqrt{2})\left(e_{\iota 1}+e_{\iota 2}\right)$. Put $e_{0}=\otimes_{I_{0}} e_{\iota}$, $\mathfrak{S}_{0}=\otimes_{I_{0}^{0} \mathfrak{S}_{\iota}}$ and $\mathfrak{U}_{0}=\otimes_{I_{0}}^{\mathfrak{c}_{0} \mathfrak{H}_{\iota}}$, where $\mathfrak{c}_{0}$ is an equivalence class which contains $\left(e_{\iota}: \iota \in I_{0}\right)$. Then $\mathfrak{2}_{0}$ is generated by $\left(\otimes_{J} E_{\iota j_{\iota}}\right) \otimes 1\left(I_{0}-J\right), j_{\iota}=1$ or 2 for $J \ll I_{0}$. Define a normalized positive linear functional $\varphi$ on $\bigodot_{I} \mathfrak{A}_{\text {. }}$ by

$$
\varphi\left(F\left(j_{\iota}^{\prime}: \iota \in J\right) \otimes\left(\otimes_{J} E_{\iota j_{\iota}}\right) \otimes 1(K)\right)=\left\{\begin{array}{cc}
1 / 2^{n} & \text { if } j_{\iota}=j_{\iota}^{\prime} \\
0 & \text { otherwise }
\end{array}\right.
$$

where $c$ is an equivalence class containing $\left(e_{\iota}: \iota \in I\right), F\left(j_{\iota}^{\prime}: \iota \in J\right)$ $=\left(\otimes_{J} E_{\iota j^{\prime}}\right) \otimes 1(K) \in \mathbb{R}\left(\mathfrak{S}_{0}\right), J \ll I_{0}, K=I_{0}-J, j_{\iota}$ or $j_{\iota}^{\prime}=1$ or 2 and $n$ is the cardinal number of $J$. Then the projection of $\varphi$ to $\mathfrak{U}_{0}$ is $\omega_{e_{0}}$ and to $\otimes_{K} \mathfrak{H}_{\text {. }}$ is $\otimes_{K} \omega_{e_{\imath}}$ for $K \ll I-\{0\}$. Denote by $\varphi(J)$ the projection of $\varphi$ to $\otimes_{J} \mathfrak{H}_{.}$for $J \ll I$. Then $\{\varphi(J): J \Subset I\}$ is a c-normal projective system, since

$$
\left\|\varphi(K)-\omega_{e(K)}\right\|=0
$$

for any $K \ll\{0\}^{c}$. However, if $0 \in J_{1} \subset J_{2}$ and $m$ is the cardinal number of $J_{2}-J_{1}$, then $\left\|\varphi\left(J_{2}\right)-\varphi\left(J_{1}\right) \otimes \varphi\left(J_{2}-J_{1}\right)\right\|=2\left(1-2^{-m}\right)$. Thus for any $J_{0} \ll I$

$$
\sup _{J_{0} \subset J_{1} \subset J_{2}}\left\|\varphi\left(J_{2}\right)-\varphi\left(J_{1}\right) \otimes \varphi\left(J_{2}-J_{1}\right)\right\|=2 .
$$

Moreover the c-normality of $\{\varphi(J): J \ll I\}$ does not follow from the condition $\left(^{*}\right)$. This is clear from the following example.

Utilizing the notations in the above example and define $\psi_{\iota}=\left(\omega_{e_{t 1}}+\omega_{e_{\mathrm{t} 2}}\right) / 2$, which is a normalized positive linear functional on $\mathcal{Q}\left(\mathfrak{S}_{\iota}\right)$. Define $\psi(J)=\bigotimes_{J} \psi_{\iota}$ for $J \ll I_{0}$. Then over $\otimes_{I_{0}}^{c_{0}} \mathbb{R}\left(\mathfrak{S}_{\iota}\right)$ the condition $\left(^{*}\right)$ holds for all $J \ll I_{0}$, 
although $\left\{\psi(J): J \Subset I_{0}\right\}$ is not c-normal.

Remark 3.4. Let $\varphi$ be a normal positive linear functional on $\otimes^{\mathrm{c}} \mathfrak{Q}_{\iota}$ with $\|\varphi\|=1$. Then for any $\varepsilon>0$ there exists $J_{0} \ll I$ such that

$$
\left|\varphi\left(A_{J_{1}} A_{J_{2}}\right)-\varphi\left(A_{J_{1}}\right) \varphi\left(A_{J_{2}}\right)\right|<\varepsilon\left\|A_{J_{1}}\right\| \cdot\left\|A_{J_{2}}\right\|
$$

for any $J_{i} \ll I(i=1,2)$ with $J_{i} \cap J_{j}=\phi(i \neq j$ and $i, j=0,1,2)$, where $A\left(J_{i}\right) \in \otimes_{J_{2}} \mathfrak{A}_{\imath}$ and $A_{J_{i}}=A\left(J_{i}\right) \otimes 1\left(J_{i}^{c}\right)(i=1,2)$. Really, by the last theorem, for any $\varepsilon>0$ there exists $J_{0} \ll I$ such that

$$
\left\|\varphi(K)-\omega_{e(K)}\right\|<\frac{\varepsilon}{3}
$$

for every $K \Subset I$ with $K \cap J_{0}=\phi$. Thus we obtain that

$$
\begin{aligned}
\mid \varphi( & \left.A_{J_{1}} A_{J_{2}}\right)-\varphi\left(A_{J_{1}}\right) \varphi\left(A_{J_{2}}\right) \mid \\
= & \left|\varphi\left(J_{1} \cup J_{2}\right)\left(A\left(J_{1}\right) \otimes A\left(J_{2}\right)\right)-\varphi\left(J_{1}\right)\left(A\left(J_{1}\right)\right) \varphi\left(J_{2}\right)\left(A\left(J_{2}\right)\right)\right| \\
\leqq & \left|\varphi\left(J_{1} \cup J_{2}\right)\left(A\left(J_{1}\right) \otimes A\left(J_{2}\right)\right)-\omega_{e\left(J_{1} \cup J_{2}\right)}\left(A\left(J_{1}\right) \otimes A\left(J_{2}\right)\right)\right| \\
& +\left|\omega_{e\left(J_{1}\right)}\left(A\left(J_{1}\right)\right)\right| \cdot\left|\left(\omega_{e\left(J_{2}\right)}-\varphi\left(J_{2}\right)\right)\left(A\left(J_{2}\right)\right)\right| \\
& +\left|\left(\omega_{e\left(J_{1}\right)}-\varphi\left(J_{1}\right)\right)\left(A\left(J_{1}\right)\right)\right| \cdot\left|\varphi\left(J_{2}\right)\left(A\left(J_{2}\right)\right)\right| \\
\leqq & \varepsilon\left\|A_{J_{1}} \mid \cdot\right\| A_{J_{2}} \| .
\end{aligned}
$$

Corollary 3.2. Let $\varphi$ be a normal positive linear functional on $\otimes^{\mathfrak{c}} \mathfrak{H}_{\text {, }}$ with $\|\varphi\|=1$ and $\{\varphi(J): J \ll I\}$ a projective system corresponding to $\varphi$.

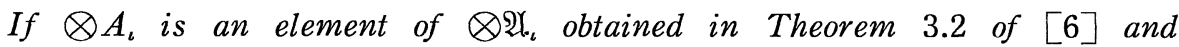
$\Pi\left(A_{\imath} e_{\iota}, e_{\iota}\right) \neq 0$ for some $\left(e_{\iota}\right) \in \mathrm{c}$ with $\left\|e_{\iota}\right\|=1$, then

$$
\varphi\left(\otimes^{c} A_{\iota}\right)=\lim _{J} \varphi(J)\left(\bigotimes_{J} A_{\iota}\right) .
$$

Proof. Since $\otimes A_{\iota}$ is non zero, there is a constant $M>1$ with

$$
M^{-1}<\left\|\otimes_{J} A_{\imath}\right\|<M
$$

for any $J \ll I$. In the case where $\varphi\left(\otimes^{c} A_{\iota}\right)$ is non zero, let $2 \varepsilon$ be smaller than its absolute value. By Lemma 3.1 we may select $I_{0} \ll I$ such that

$$
\left\|\varphi-\varphi(J) \otimes \omega_{e\left(J^{c}\right)}\right\|<\frac{\varepsilon}{M}
$$


for every $J \ll I$ with $I_{0} \subset J$. Hence we have

$$
\left|\varphi\left(\otimes^{c} A_{\iota}\right)-\varphi(J)(A(J)) \omega_{e\left(J^{c}\right)}\left(A\left(J^{c}\right)\right)\right|<\varepsilon,
$$

where $A(J)=\otimes_{J} A_{\iota}$ and $A\left(J^{c}\right)=\bigotimes_{J^{c}}^{c} A_{\iota}$. Thus

$$
\left|\varphi(J)(A(J)) \omega_{e\left(J^{c}\right)}\left(A\left(J^{c}\right)\right)\right| \geqq \varepsilon .
$$

From this we find that for $\varepsilon>0$ in the above there exists $I_{1} \ll I$ with $I_{0} \subset I_{1}$ such that

$$
\left|\omega_{e\left(J^{c}\right)}\left(A\left(J^{c}\right)\right)-1\right|<\varepsilon
$$

for every $J \ll I$ with $I_{1} \subset J$. Consequently

$$
\begin{aligned}
&\left|\varphi\left(\otimes^{c} A_{\iota}\right)-\varphi(J)(A(J))\right| \\
& \leqq\left|\varphi\left(\otimes^{c} A_{\iota}\right)-\varphi(J)(A(J)) \omega_{e\left(J^{c}\right)}\left(A\left(J^{c}\right)\right)\right| \\
& \quad+\left|\varphi(J)(A(J))\left(\omega_{e\left(J^{c}\right)}\left(A\left(J^{c}\right)\right)-1\right)\right| \\
& \leqq(1+M) \varepsilon .
\end{aligned}
$$

Next consider the case where $\varphi\left(\otimes^{c} A_{\imath}\right)=0$. According to Corollary 4.1 in [6], we have $\omega_{e(I)}\left(\otimes^{c} A_{\iota}\right)=\Pi \omega_{e_{\iota}}\left(A_{\iota}\right)$. Since $\Pi\left(A_{\iota} e_{\iota}, e_{\iota}\right) \neq 0$ for some $\left(e_{\iota}\right) \in \mathrm{c}$, we have $\omega_{e(I)}\left(\otimes^{c} A_{\imath}\right) \neq 0$ and hence for any $\varepsilon>0$ there exists $J_{0} \ll I$ such that

$$
\left|\omega_{e\left(J^{c}\right)}\left(A\left(J^{c}\right)\right)-1\right|<\varepsilon
$$

for every $J \Subset I$ with $J_{0} \subset J$. Thus by the above (*) we obtain

$$
\begin{aligned}
|\varphi(J)(A(J))| \leqq & \left|\varphi(J)(A(J))\left(1-\omega_{e\left(J^{c}\right)}\left(A\left(J^{c}\right)\right)\right)\right| \\
& +\left|\varphi(J)(A(J)) \omega_{e\left(J^{c}\right)}\left(A\left(J^{c}\right)\right)\right| \\
\leqq & (M+1) \varepsilon .
\end{aligned}
$$

Remark 3.5. This corollary tells us that if $\|\varphi\|=1$ and $\otimes^{c} A_{\iota} \neq 0$, then

$$
\lim _{J \subset I} \varphi\left(J^{c}\right)\left(A\left(J^{c}\right)\right)=1
$$

Indeed, by Theorem 3.1 there exists $J_{1} \ll I$ such that

$$
\left\|\varphi(K)-\omega_{e(K)}\right\|<\frac{\varepsilon}{M}
$$


for $K \ll I$ with $K \cap J_{1}=\phi$. If $\otimes^{c} A_{\iota} \neq 0$, then there exists $\left(e_{\iota}\right) \in \mathrm{c}$ such that $\left\|e_{\iota}\right\|=1$ and $\Pi\left(A_{\iota} e_{\iota}, e_{\iota}\right) \neq 0$ and hence by the formula (**) we have

$$
\left|\omega_{e\left(J^{c}\right)}\left(A\left(J^{c}\right)\right)-1\right|<\varepsilon
$$

for $J \ll I$ with $J_{0} \subset J$. Therefore by Corollary 3.2, there exists $J_{2} \ll I$ with $J_{0} \cup J_{1} \subset J_{2}$ such that

$$
\left|\omega_{e\left(J^{c}\right)}\left(A\left(J^{c}\right)\right)-\omega_{e(K)}(A(K))\right|<\varepsilon
$$

and

$$
\left|\varphi\left(J^{c}\right)\left(A\left(J^{c}\right)\right)-\varphi(K)(A(K))\right|<\varepsilon
$$

for every $J \ll I$ with $J_{2} \subset J$ and for some $K \ll J^{c}$. Thus we get

$$
\begin{aligned}
\left|\varphi\left(J^{c}\right)\left(A\left(J^{c}\right)\right)-1\right| \leqq & \left|\varphi\left(J^{c}\right)\left(A\left(J^{c}\right)\right)-\varphi(K)(A(K))\right| \\
& +\left|\varphi(K)(A(K))-\omega_{e(K)}(A(K))\right| \\
& +\left|\omega_{e(K)}(A(K))-\omega_{e\left(J^{c}\right)}\left(A\left(J^{c}\right)\right)\right| \\
& +\left|\omega_{e\left(J^{c}\right)}\left(A\left(J^{c}\right)\right)-1\right| \\
& <4 \varepsilon .
\end{aligned}
$$

Corollary 3.3. Given a normal positive linear functional $\varphi$ on $\otimes \mathcal{H}_{\imath}$ with $\varphi(1)=\varphi(P(\mathfrak{c}))$, and a projective system $\{\varphi(J): J \Subset I\}$ corresponding to the restriction $\varphi \mid \otimes \mathfrak{N}_{\text {. }}$. Let $G$ and $G(J)$ be the carrier of $\varphi$ and $\varphi(J)$ respectively. Denote $G_{J}=G(J) \otimes E\left(\left(\otimes_{J^{r}} 2 l_{L}\right)^{\prime}, e\left(J^{c}\right)\right)$ and $G_{J}^{\prime}=G(J) \otimes 1\left(J^{c}\right)$. Then

$$
G \leqq \lim _{J} G_{J} P(\mathfrak{c})=\lim _{J} G_{J}^{\prime} P(\mathfrak{c}) .
$$

Proof. It is easily seen that $\left\{G_{J}^{\prime}: J \Subset I\right\}$ is a monotone decreasing Cauchy net in the weak topology, whose limit is a projection $G_{0}$. Hence for any $\varepsilon>0$ and for any $x=\left(\otimes_{K_{1}} x_{\imath}\right) \otimes e\left(K_{1}^{c}\right)$ and $y=\left(\otimes_{K_{2}} y_{\imath}\right) \otimes e\left(K_{2}^{c}\right)$ with $K_{i} \ll I(i=1,2)$ there exists $I_{0} \ll I$ such that

$$
\left|\left(\left(G_{K}^{\prime}-G_{0}\right) x, y\right)\right|<\varepsilon
$$

for any $K \ll I$ with $I_{0} \subset K$. Denote $I_{1}=K_{1} \cup K_{2} \cup I_{0}$. Then for any $J \ll I$ with $I_{1} \subset J$ we have $G_{J} x=G_{J}^{\prime} x$ and $G_{J} y=G_{J}^{\prime} y$, and hence for any $J \ll I$ with $I_{1} \subset J$ 


$$
\left|\left(\left(G_{J}-G_{0}\right) x, y\right)\right|<\varepsilon
$$

Since the subset of vectors of the above form is total in $\otimes^{\prime} \mathfrak{S}_{\iota}$, the similar evaluation holds for every vectors in $\otimes^{c} \mathfrak{S}_{\imath}$, which shows that $\left\{G_{J} P_{\mathrm{c}}: J \ll I\right\}$ and $\left\{G_{J}^{\prime} P_{\mathrm{c}}: J \ll I\right\}$ have the same limit, and hence so do $\left\{G_{J} P(\mathfrak{c}): J \ll I\right\}$ and $\left\{G_{J}^{\prime} P(\mathfrak{c}): J \Subset I\right\}$. Inequality is obvious from $G \leqq G_{J}^{\prime}$ by Lemma 3.2.

Q.E.D.

If $\varphi$ is of the form of tensor product, then the carrier is calculated in Corollary 4.2 in $[6]$, which shows that $G=\lim _{J} G_{J}^{\prime} P(c)$. In general, it is not obvious whether equality holds or not.

Remark 3.6. Even if a uniformly convergent sequence or net $\left\{\varphi_{\lambda}: \lambda \in \Lambda\right\}$ of normal positive linear functionals $\varphi_{\lambda}, \lambda \in \Lambda$ with $\varphi_{\lambda}(1)=1$ is given, we cannot necessarily conclude that the carrier of the limit functional may be approximated by the carriers of $\varphi_{\lambda}, \lambda \in \Lambda$ in the weak topology. Indeed, let $\mathfrak{S}$ be a two-dimensional Hilbert space and $\left\{e_{1}, e_{2}\right\}$ its orthonormal base. Define $\varphi=\omega_{e_{1}}$ and $\varphi_{\varepsilon}=(1-\varepsilon) \omega_{e_{1}}+\varepsilon \omega_{e_{2}}$ for $0<\varepsilon<1$. Then $\varphi_{\varepsilon}$ and $\varphi$ are normal positive linear functionals on $\mathscr{L}(\mathfrak{E})$ such that $\varphi_{\varepsilon}(1)=\varphi(1)=1$, and their carriers are the identity 1 and the projection $E$ onto $\left\{\alpha e_{1}: \alpha \in C\right\}$ respectively. Hence we have $\left((1-E) e_{2}, e_{2}\right)=1$, while $\left\|\varphi-\varphi_{\varepsilon}\right\|=2 \varepsilon$.

Corollary 3.4. Let $\mathfrak{A}_{\iota}$ be a finite factor on $\mathfrak{S}_{\iota}$ of at least two dimension for $\iota \in I$, each of which has the coupling constant $C_{\imath}$ with $\sum \max \left\{1-C_{\iota}, 0\right\}<+\infty$. Let $x_{\iota} \in \mathfrak{S}_{\imath}$ be a characteristic vector of a normal trace $\varphi_{\iota}$ on $\mathfrak{U}_{\iota}$ with $\varphi_{\iota}(1)=1$. Then $\otimes^{(c)} \mathfrak{U}_{\imath}$ is a finite factor if and only if $P(\mathfrak{c})\left(\otimes x_{\imath}\right)=\otimes x_{\iota}$.

Proof. By Theorem 4.2 and Corollary 4.2 in $[6] \otimes \varphi_{\iota}$ is a normal trace on $\otimes \mathfrak{U}_{\iota}$ whose carrier is $P(\mathfrak{c})$. Hence $\otimes^{(c)} \mathfrak{Q}_{\iota}$ is a finite factor, if $P(\mathrm{c})\left(\otimes x_{\imath}\right)=\otimes x_{\iota}$.

On the contrary, if $\otimes^{\left(c^{\prime}\right)} \mathfrak{Q}_{\iota}$ is a finite factor, then there is a normal trace $\varphi$ on $\otimes \mathfrak{A}_{\iota}$ with $\varphi\left(P\left(\mathfrak{c}^{\prime}\right)\right)=\varphi(1)=1$. Let $\left(e_{\iota}\right) \in \mathfrak{c}^{\prime}$ and $\phi$ be the restriction of $\varphi$ to $\otimes^{\prime} \mathfrak{A}_{\iota}$. Then the family $\{\psi(J): J \Subset I\}$ of projections of $\phi$ to $\otimes_{J} \mathfrak{A}_{\imath}$ is a projective system and for any $\varepsilon>0$ there exists $J_{0} \ll I$ such that 


$$
\left\|\psi-\psi(J) \otimes \omega_{e\left(J^{c}\right)}\right\|<\varepsilon
$$

for any $J \Subset I$ with $J_{0} \subset J$. Since $\phi(J)$ is a normal trace on $\otimes_{J} \mathfrak{A}_{\iota}$, we have $\phi(J)=\otimes_{J} \varphi_{\iota}$ and hence

$$
\varphi\left(\prod_{K} \bar{A}_{\iota}\right)=\psi\left(\left(\bigotimes_{K} A_{\iota}\right) \otimes\left(\bigotimes_{K^{c}}^{c^{\prime}} 1\right)\right)=\prod_{K} \varphi_{\iota}\left(A_{\iota}\right)
$$

for any $K \ll I$ and $A_{\iota} \in \mathfrak{H}_{\iota}$. Thus $\varphi=\otimes \varphi_{\iota}$ by Theorem 4.2 in $[6]$. We conclude that $P\left(\mathfrak{c}^{\prime}\right)=P(\mathfrak{c})$.

Q.E.D.

As we know the circumstances of the finite part of a complete infinite tensor product of finite factors in the last corollary, we can develop our study in more detail in the following section.

\section{§4. Decomposition of a Finite Normal Trace on Some $\otimes \mathscr{N}_{\imath}$}

If $\mathfrak{A}$. and $\mathfrak{A}^{\prime}$ are finite, then the coupling operator $C$ of $\mathfrak{A}$ satisfies that $0<C(\zeta)<+\infty \nu$-locally almost everywhere on $Z$. In case where $\mathfrak{A}$. is finite and $\mathfrak{2} !$ is not finite, decompose $\mathfrak{V}^{\prime}$ into a finite $\mathfrak{V}_{G}^{\prime}$ and a properly infinite $\mathfrak{A}_{1-G}^{\prime}$ with $G \in\left(\mathfrak{A} \cap \mathfrak{A}^{\prime}\right)^{p}$ and define $C=C_{G}$ on $G \mathfrak{C}$ and $+\infty$ on $(1-G) \mathfrak{S}$ where $C_{G}$ is the coupling operator of $\mathfrak{A}_{G}$, which we also call the coupling operator of $\mathfrak{U}$.

Let $Z$ be a locally compact space and let $\nu$ and $\mu$ be positive Radon measures on $Z$ such that $\mu$ is absolutely continuous with respect to $\nu$, that is, $d \mu=f d \nu$ by a locally $\nu$-integrable $f$ with $0 \leqq f(\zeta)<+\infty$ for $\zeta \in Z$. Let $Y=\{\zeta \in Z: f(\zeta)>0\}$. Then $Y$ is $\nu$-measurable and the restrictions $\mu \mid Y$ and $\nu \mid Y$ of $\mu$ and $\nu$ to $Y$ are equivalent. Thus

$$
\int_{Z}^{\oplus} \mathfrak{E}(\zeta) d \mu(\zeta) \quad \text { and } \quad \int_{Y}^{\oplus} \mathfrak{E}(\zeta) d \mu(\zeta)
$$

are isomorphic,

$$
\int_{Y}^{\oplus} \mathfrak{S}(\zeta) d \mu(\zeta) \quad \text { and } \quad \int_{Y}^{\oplus} \mathfrak{S}(\zeta) d \nu(\zeta)
$$

are isomorphic and the last one is a subspace of

$$
\int_{Z}^{\oplus} \mathfrak{S}(\zeta) d \nu(\zeta)
$$


Therefore an isomorphism of

$$
\int_{Z}^{\oplus} \mathfrak{g}(\zeta) d \mu(\zeta) \quad \text { onto } \quad \int_{Y}^{\oplus} \mathfrak{g}(\zeta) d \nu(\zeta)
$$

maps a square $\mu$-integrable field $\zeta \rightarrow x(\zeta)$ to a square $\nu$-integrable field $\zeta \rightarrow \sqrt{f(\zeta)} x(\zeta)$. Let $\zeta \rightarrow A(\zeta)$ denote a $\mu$-measurable field of operators. Define $B(\zeta)=A(\zeta)$ for $\zeta \in Y$ and $B(\zeta)=0$ for $\zeta \in Z-Y$. Then $\zeta \rightarrow B(\zeta)$ on $Y$ is $\mu \mid Y$-measurable and hence it is $\nu \mid Y$-measurable. Since $Y$ is $\nu$-measurable, $\zeta \rightarrow B(\zeta)$ is a $\nu$-measurable field on $Z$. Thus for any $\mu$ measurable field $\zeta \rightarrow A(\zeta)$ we can obtain a $\nu$-measurable field $\zeta \rightarrow B(\zeta)$ such that

$$
\int_{Z}^{\oplus} A(\zeta) d \mu(\zeta)=\int_{Z}^{\oplus} B(\zeta) d \mu(\zeta)
$$

Consequently we may consider the set of $\mu$-measurable fields of operators is contained in the set of $\nu$-measurable fields of operators by the modification of a $\mu$-null set.

Lemma 4.1. Let $Z$ be a locally compact space, $\nu$ and $\mu$ positive Radon measures on $Z$ such that $\mu$ is absolutely continuous with respect to $\nu$,

$$
\mathfrak{Q}=\int_{Z}^{\oplus} \mathfrak{Q}(\zeta) d \nu(\zeta), \quad \mathfrak{U}=\int_{Z}^{\oplus} \mathfrak{A}(\zeta) d \nu(\zeta)
$$

and $\zeta \rightarrow \varphi_{\zeta}$ a field of normal traces. If the field $\zeta \rightarrow \varphi_{\zeta}$ is $\nu$-measurable, then it is $\mu$-measurable.

Proof. If $\zeta \rightarrow A(\zeta)$ is a $\mu$-measurable field of operators, then there is a $\nu$-measurable field $\zeta \rightarrow B(\zeta)$ such that

$$
\int_{Z}^{\oplus} A(\zeta) d \mu(\zeta)=\int_{Z}^{\oplus} B(\zeta) d \mu(\zeta)
$$

By the hypothesis that $\zeta \rightarrow \varphi_{\zeta}(B(\zeta))$ is $\nu$-measurable, we have that it is $\mu$-measurable. Since $Y$ is $\mu$-measurable, the field $\zeta \rightarrow \varphi_{\zeta}(A(\zeta))$ is $\mu$ measurable, which implies the desired result.

Q.E.D.

In the following, the notations $Z_{\iota}, \nu_{\iota}, \zeta_{\iota}, \mathfrak{S}_{\iota}\left(\zeta_{\iota}\right), \mathfrak{N}_{\iota}\left(\zeta_{\iota}\right), \mathfrak{S}_{\iota}, \mathfrak{Z}_{\iota}$ and $\mathfrak{N}_{\iota}$ are used as in the introduction and a series of lemmas is stated, assuming 
some of the following seven conditions:

(A1) $I$ is countable;

(A2) $Z_{\iota}$ is compact;

(A3) $Z_{\iota}$ satisfies the second axiom of countability;

(A4) $\quad\left\|\nu_{\iota}\right\|=1$;

(A5) $\mathfrak{U}_{\iota}$ is finite;

(A6) $\mathfrak{B}_{\iota}$ is the center of $\mathfrak{H}_{\iota}$; and

(A7) $\sum\left\|\left(1-C_{\iota}\right)^{+}\right\|<+\infty$, where $C_{\iota}$ is the coupling operator of $\mathfrak{A}_{\iota}$.

Among these conditions, (A1) is needed to construct measurable fields of an incomplete infinite tensor product of Hilbert spaces and of von Neumann algebras, because the method we will employ in this paper is developed under the hypothesis that each stalk is separable. (A2) is needed to make $Z=\Pi Z$ ، locally compact, because the reduction theory on a completely regular space which is not locally compact is not yet prepared. From (A3) and (A5), we know that stalks of a von Neumann algebra are finite locally almost everywhere on $Z$. By (A4) we can avoid the complexity of the product measure $\otimes \nu_{\imath}$. The boundedness of measure, (A4), implies the countable decomposability of the von Neumann algebra. The countable decomposability also follows from (A3). (A6) is needed to ensure the factor decompositions. (A7) is the condition that the infinite tensor product of finite normal traces on some stalks of finite von Neumann algebras can be defined on the infinite tensor product of respective stalks of von Neumann algebras.

The following notations are sometimes used:

Let $A$ be the set of all functions $i$ defined on $I$ and having the values in $z^{\prime}$ such that $i(\iota)=0$ on $I-J$ with $J \Subset I$. Denote $Z=\Pi Z$ a and $Z(J)=\Pi_{J} Z_{\iota}$ for $J \subset I$. Let $\mathfrak{P}$ be the set of all continuous functions of the form $g=\left(\otimes_{J} g_{\imath}\right) \otimes 1\left(J^{c}\right)$ defined on a compact space $Z$ for every $J \ll I$, where $g_{\iota} \in C\left(Z_{\iota}\right)$ and $1\left(J^{c}\right)$ is a constant 1 function on $Z\left(J^{c}\right)$. Then the *-algebra $C_{0}(Z)$ generated algebraically by $\mathfrak{P}$ is uniformly dense in $C(Z)$ by the Stone-Weierstrass theorem.

Let $\zeta_{\iota} \rightarrow \mathfrak{S}_{\iota}\left(\zeta_{\iota}\right)$ be a $\nu_{\iota}$-measurable field of Hilbert spaces on $Z_{\iota}$ and let $\mathbb{S}_{\iota}$ be a vector subspace of $\Pi_{z_{\iota}} \mathscr{S}_{\iota}\left(\zeta_{\iota}\right)$ defining the $\nu_{\iota}$-measurable field and 


$$
\mathfrak{Q}_{\iota}=\int_{Z_{\iota}}^{\oplus} \mathfrak{Q}_{\iota}\left(\zeta_{\iota}\right) d \nu_{\iota}\left(\zeta_{\iota}\right)
$$

for every $\iota \in I$. The subset $\left\{x_{i(\iota), \iota} ; i \in \Lambda\right\}$ of $\mathscr{S}_{\iota}$ is a fundamental sequence of $\nu_{\iota}$-measurable field $\zeta_{\iota} \rightarrow x_{i(\iota), \iota}\left(\zeta_{\iota}\right)$ of vectors in $\mathfrak{E}_{\iota}\left(\zeta_{\iota}\right)$, for which we may assume that $\left\|x_{i(\iota), \iota}\left(\zeta_{\iota}\right)\right\|=1$ for all $\zeta_{\iota}$ by multiplying a suitable $\nu_{\iota}$ measurable function. Denote by $\mathrm{c}$ or $\mathrm{c}(\zeta)$ for $\zeta=\left(\zeta_{\iota}\right)$ in $Z$ an equivalence class of $C_{0}$-sequences for $\mathfrak{C}_{\iota}$ 's and $\mathfrak{C}_{\iota}\left(\zeta_{\iota}\right)$ 's for $\iota \in I$ respectively. Suppose $\left(x_{0, \iota}\right) \in \mathfrak{c}$ and $\left(x_{0, \iota}\left(\zeta_{\iota}\right)\right) \in \mathfrak{c}(\zeta)$. Put $x_{i, \iota}=x_{i(\iota), \iota}, x_{i, \iota}\left(\zeta_{\iota}\right)=x_{i(\iota), \iota}\left(\zeta_{\iota}\right)$, $(\otimes x)_{i}=\otimes x_{i, \iota}$ and $(\otimes x)_{i}(\zeta)=\otimes x_{i, \iota}\left(\zeta_{\iota}\right)$ in the following of this paper. Then $\zeta=\left(\zeta_{\iota}\right) \rightarrow \otimes^{c(\zeta)} \mathfrak{S}_{\iota}\left(\zeta_{\iota}\right)$ is a field of Hilbert spaces on $Z$ and $\zeta \rightarrow(\otimes x)_{i}(\zeta)$ is a field of vectors on $Z$ such that

$$
\left((\otimes x)_{i}(\zeta),(\otimes x)_{j}(\zeta)\right)=\Pi\left(x_{i, \iota}\left(\zeta_{\iota}\right), x_{j, \iota}\left(\zeta_{\iota}\right)\right)
$$

is $\otimes \nu_{\imath}$-measurable and the set of $(\otimes x)_{i}(\zeta)$ with $i \in \Lambda$ forms a total subset in $\otimes^{c(\zeta)} \mathfrak{S}_{\iota}\left(\zeta_{\iota}\right)$. Denote by $\otimes^{c}\left(\mathcal{S}_{\iota}\right.$ the subset of $z(\zeta)$ in $\Pi_{z} \otimes^{c(\zeta)} \mathfrak{K}_{\iota}\left(\zeta_{\iota}\right)$ such that $\left(z(\zeta),(\otimes x)_{i}(\zeta)\right)$ is $\otimes \nu_{\imath}$-measurable for all $i \in \Lambda$. Then, utilizing this $\otimes^{c} \mathscr{F}_{\iota}$, we can obtain a $\otimes \nu_{\iota}$-measurable field

$$
\zeta=\left(\zeta_{\imath}\right) \rightarrow \otimes^{c(\zeta)} \mathfrak{S}_{\imath}\left(\zeta_{\imath}\right)
$$

of Hilbert spaces on $Z$.

Lemma 4.2. Assume (A1), (A2) and (A4). If $\left(x_{0, \imath}\right) \in \mathrm{c}$ and $\left(x_{0, \iota}\left(\zeta_{\iota}\right)\right) \in \mathfrak{c}(\zeta)$ for $\zeta=\left(\zeta_{\iota}\right)$, then $\otimes \mathfrak{S}_{\imath}$ is isomorphic to

$$
\int_{Z}^{\oplus} \otimes^{c(\zeta)} \mathfrak{Q}_{\iota}\left(\zeta_{\iota}\right) d\left(\otimes \nu_{\iota}\right)(\zeta)
$$

Proof. As $\left(x_{i, \imath}\right) \in \mathrm{c}$ and

$$
x_{i, \iota}=\int_{Z_{\iota}}^{\oplus} x_{i, \iota}\left(\zeta_{\iota}\right) d \nu_{\iota}\left(\zeta_{\iota}\right),
$$

$\zeta_{\iota} \rightarrow x_{i, \iota}\left(\zeta_{\iota}\right)$ is $\nu_{\iota}$-measurable and $\left(x_{i, \iota}\left(\zeta_{\iota}\right)\right) \in \mathfrak{c}(\zeta)$. Since the set of all finite linear combinations of $g_{\iota} x_{i, \iota}$ for every $g_{\iota} \in C\left(Z_{\iota}\right)$ and for every $i \in \Lambda$ is dense in $\mathfrak{S}_{\iota}$ for every $\iota \in I$, it follows that the set of all finite linear combinations on $g(\otimes x)_{i}=\otimes g_{\imath} x_{i, \iota}$ for every $g=\otimes g_{\imath}$ in $\mathfrak{P}$ and for every $i \in \Lambda$ is dense in $\otimes \mathfrak{S}_{\imath}$. On the other hand, if $g=\otimes g_{\imath}$ belongs to $\mathfrak{H}$, 
then $g(\zeta)(\otimes x)_{i}(\zeta)=\otimes g_{\iota}\left(\zeta_{\iota}\right) x_{i, \iota}\left(\zeta_{\iota}\right)$ and $\zeta \rightarrow g(\zeta)(\otimes x)_{i}(\zeta)$ is $\otimes \nu_{\iota}$-measurable. Since $\mathfrak{P}$ is total in $C(Z)$, the set of all finite linear combinations of these fields $\zeta \rightarrow g(\zeta)(\otimes x)_{i}(\zeta)$ for $g \in \mathfrak{P}$ and $i \in \Lambda$ is a dense linear subset of

$$
\int_{Z}^{\oplus} \otimes^{(\zeta)} \mathfrak{G}_{\iota}\left(\zeta_{\iota}\right) d\left(\otimes \nu_{\imath}\right)(\zeta) .
$$

Since for any $g_{i}=\otimes g_{i, \iota} \in \mathfrak{P}$

$$
\begin{aligned}
\left\|\sum_{i} g_{i}(\otimes x)_{i}\right\|^{2} & =\sum_{i, j}\left(g_{i}(\otimes x)_{i}, g_{j}(\otimes x)_{j}\right) \\
& =\sum_{i, j}\left(\otimes g_{i, \iota} x_{i, \iota}, \otimes g_{j, \iota} x_{j, \iota}\right) \\
& =\sum_{i, j} \Pi\left(g_{i, \iota} x_{i, \iota}, g_{j, \iota} x_{j, \iota}\right) \\
& =\sum_{i, j} \Pi \int_{Z_{\iota}}\left(g_{i, \iota}\left(\zeta_{\iota}\right) x_{i, \iota}\left(\zeta_{\iota}\right), g_{j, \iota}\left(\zeta_{\iota}\right) x_{j, \iota}\left(\zeta_{\iota}\right)\right) d \nu_{\iota}\left(\zeta_{\iota}\right) \\
& =\sum_{i, j} \int_{Z} \Pi\left(g_{i, \iota}\left(\zeta_{\iota}\right) x_{i, \iota}\left(\zeta_{\iota}\right), g_{j, \iota}\left(\zeta_{\iota}\right) x_{j, \iota}\left(\zeta_{\iota}\right)\right) d\left(\otimes \nu_{\iota}\right)(\zeta) \\
& =\sum_{i, j} \int_{Z}\left(g_{i}(\zeta)(\otimes x)_{i}(\zeta), g_{j}(\zeta)(\otimes x)_{j}(\zeta)\right) d\left(\otimes \nu_{\iota}\right)(\zeta) \\
& =\int_{Z}\left\|\sum_{i} g_{i}(\zeta)(\otimes x)_{i}(\zeta)\right\|^{2} d\left(\otimes \nu_{\iota}\right)(\zeta),
\end{aligned}
$$

the similar equality holds for any $g_{i} \in C_{0}(Z)$,

$$
\left\|\sum_{i} g_{i}(\otimes x)_{i}\right\|^{2}=\int_{Z}\left\|\sum_{i} g_{i}(\zeta)(\otimes x)_{i}(\zeta)\right\|^{2} d\left(\otimes \nu_{\imath}\right)(\zeta) .
$$

It follows that the linear mapping which transforms $\sum_{i} g_{i}(\otimes x)_{i}$ to the field $\zeta \rightarrow \sum_{i} g_{i}(\zeta)(\otimes x)_{i}(\zeta)$ is extended to an isometric linear mapping $\theta$ of Q) $\mathscr{S}_{2}$ onto

$$
\int_{Z}^{\oplus} \otimes^{c(\zeta)} \xi_{\iota}\left(\zeta_{\iota}\right) d\left(\otimes \nu_{\iota}\right)(\zeta) .
$$

Q.E.D.

Through this isomorphism $\theta$ we shall identify $\otimes^{\mathfrak{c}} \mathfrak{S}_{\imath}$ and

$$
\int_{Z}^{\oplus} \otimes^{c(\zeta)} \mathscr{S}_{\iota}\left(\zeta_{\iota}\right) d\left(\otimes \nu_{\imath}\right)(\zeta)
$$


in the following.

Remark 4.1. In the last lemma it should be noted that the assumption (A2) is not necessary if $I$ is finite. By the above identification, if

$$
x_{\iota}=\int_{Z}^{\oplus} x_{\iota}\left(\zeta_{\iota}\right) d \nu_{\iota}\left(\zeta_{\iota}\right)
$$

with $\left\|x_{\iota}\left(\zeta_{\iota}\right)\right\|=1$, then $\otimes x_{\iota}$ is considered to be equal to

$$
\int_{Z}^{\oplus} \otimes x_{\iota}\left(\zeta_{\iota}\right) d\left(\otimes \nu_{\iota}\right)(\zeta)
$$

If a field $\zeta_{\iota} \rightarrow \mathscr{S}_{\iota}\left(\zeta_{\iota}\right)$ of Hilbert spaces is given, we shall denote the identity on $\mathscr{S}_{\iota}\left(\zeta_{\iota}\right)$ by $1\left(\zeta_{\iota}\right)$. Let $J \ll I$. If $\zeta_{\iota} \rightarrow A_{\iota}\left(\zeta_{\iota}\right)$ with $A_{\iota}\left(\zeta_{\iota}\right) \in \mathbb{Q}\left(\mathscr{S}_{\iota}\left(\zeta_{\iota}\right)\right)$ is a $\nu_{\iota}$-measurable field of operators on $Z_{\iota}$ and $A_{\iota}\left(\zeta_{\iota}\right)=1\left(\zeta_{\iota}\right)$ for $\iota \notin J$, then

$$
\zeta=\left(\zeta_{\iota}\right) \rightarrow A(\zeta)=\otimes A_{\iota}\left(\zeta_{\iota}\right)
$$

is a field of operators on $Z$ for $\otimes \mathfrak{S}_{\iota}\left(\zeta_{\iota}\right)$. If $\zeta=\left(\zeta_{\iota}\right) \rightarrow z(\zeta)$ with $z(\zeta) \in \otimes^{\mathrm{c}(\zeta)} \mathfrak{S}_{\iota}\left(\zeta_{\iota}\right)$ is a $\otimes \nu_{\iota}$-measurable field, then

$$
\left(A(\zeta) z(\zeta),(\otimes x)_{i}(\zeta)\right)=\left(z(\zeta), \otimes A_{\iota}\left(\zeta_{\iota}\right) * x_{i, \iota}\left(\zeta_{\iota}\right)\right)
$$

is $\otimes \nu_{\iota}$-measurable for all $\zeta=\left(\zeta_{\imath}\right) \rightarrow(\otimes x)_{i}(\zeta)$ with $i \in \Lambda$. Hence $\zeta \rightarrow A_{J}(\zeta)$ $=\otimes^{c(\zeta)} A_{\iota}\left(\zeta_{\iota}\right)$ is a $\otimes \nu_{\iota}$-measurable field of operators on $Z$.

Let

$$
A_{\iota}=\int_{Z_{\iota}}^{\oplus} A_{\iota}\left(\zeta_{\iota}\right) d \nu_{\iota}\left(\zeta_{\iota}\right)
$$

Then the field $\zeta_{\iota} \rightarrow A_{\iota}\left(\zeta_{\iota}\right)$ is $\nu_{\iota}$-measurable and $\nu_{\iota}$-essentially bounded on $Z_{\iota}$, and hence $\zeta \rightarrow A_{J}(\zeta)$ is also $\otimes \nu_{\iota}$-measurable and $\otimes \nu_{\iota}$-essentially bounded for $J \ll I$. If

$$
x_{i, \iota}=\int_{Z_{\iota}}^{\oplus} x_{i, \iota}\left(\zeta_{\iota}\right) d \nu_{\iota}\left(\zeta_{\iota}\right)
$$

then

$$
f_{i, \iota} x_{i, \iota}=\int_{Z}^{\oplus} f_{i, \iota}\left(\zeta_{\iota}\right) x_{i, \iota}\left(\zeta_{\iota}\right) d \nu_{\iota}\left(\zeta_{\iota}\right)
$$

for any $f_{i, \iota} \in C\left(Z_{\iota}\right)$ and hence 


$$
A_{\iota}\left(f_{i, \iota} x_{i, \iota}\right)=\int_{Z_{\iota}}^{\oplus} A_{\iota}\left(\zeta_{\iota}\right) f_{i, \iota}\left(\zeta_{\iota}\right) x_{i, \iota}\left(\zeta_{\iota}\right) d \nu_{\iota}\left(\zeta_{\iota}\right)
$$

Here we denote $A_{J}=\otimes^{c} A_{\iota}$ with $A_{\iota}=1$ for $\iota \notin J$. Then by Lemma 4.2

$$
\begin{aligned}
A_{J}\left(\sum_{i} f_{i}(\otimes x)_{i}\right) & =A_{J} \sum_{i} \otimes f_{i, \iota} x_{i, \iota} \\
& =\sum_{i} \otimes A_{\iota}\left(f_{i, \iota} x_{i, \iota}\right) \\
& =\sum_{i} \int_{Z}^{\oplus} \otimes A_{\iota}\left(\zeta_{\iota}\right) f_{i, \iota}\left(\zeta_{\iota}\right) x_{i, \iota}\left(\zeta_{\iota}\right) d\left(\otimes \nu_{\iota}\right)(\zeta) \\
& =\sum_{i} \int_{Z}^{\oplus} A_{J}(\zeta)\left(\otimes f_{i, \iota}(\zeta) x_{i, \iota}\left(\zeta_{\iota}\right)\right) d\left(\otimes \nu_{\iota}\right)(\zeta) \\
& =\sum_{i} \int_{Z}^{\oplus} A_{J}(\zeta) f_{i}(\zeta)(\otimes x)_{i}(\zeta) d\left(\otimes \nu_{\iota}\right)(\zeta) \\
& =\left(\int_{Z}^{\oplus} A_{J}(\zeta) d\left(\otimes \nu_{\iota}\right)(\zeta)\right)\left(\sum_{i} f_{i}(\otimes x)_{i}\right)
\end{aligned}
$$

for any $f_{i}=\otimes f_{i, \iota}$ in $\mathfrak{P}$. Similar calculation holds for every element in $C_{0}(Z)$ instead of in $\mathfrak{F}$ by a slight modification of notations. Consequently the following lemma is obtained.

Lemma 4.3. If

$$
A_{\iota}=\int_{Z_{\iota}}^{\oplus} A_{\iota}\left(\zeta_{\iota}\right) d \nu_{\iota}\left(\zeta_{\iota}\right)
$$

for $\iota \in 1$, then

$$
\otimes^{c} A_{\iota}=\int_{Z}^{\oplus} \otimes^{\mathrm{c}(\zeta)} A_{\iota}\left(\zeta_{\iota}\right) d\left(\otimes \nu_{\iota}\right)(\zeta)
$$

for $\zeta=\left(\zeta_{\iota}\right) \in Z$, where $A_{\iota}=1$ and $A_{\iota}\left(\zeta_{\iota}\right)=1$ for $\iota \notin J$ and $J \Subset I$.

From this lemma we have the following.

Lemma 4.4. Assume (A1), (A2), (A3) and (A4). If

$$
\mathfrak{A}_{\iota}=\int_{Z_{\iota}}^{\oplus} \mathfrak{N}_{\iota}\left(\zeta_{\iota}\right) d \nu_{\iota}\left(\zeta_{\iota}\right)
$$

for $\iota \in I$, then

$$
\otimes^{\mathfrak{c} \mathfrak{A}_{\iota}}=\int_{Z}^{\oplus} \otimes^{\mathfrak{c}(\zeta) \mathfrak{N}_{.}}\left(\zeta_{\iota}\right) d\left(\otimes \nu_{\iota}\right)(\zeta)
$$


for $\zeta=\left(\zeta_{\iota}\right) \in Z$.

Proof. Since

$$
\mathfrak{U}_{\iota}=\int_{Z_{\iota}}^{\oplus} \mathfrak{U}_{\iota}\left(\zeta_{\iota}\right) d \nu_{\iota}\left(\zeta_{\iota}\right)
$$

there is a sequence $\zeta_{\iota} \rightarrow A_{i(\iota), \iota}\left(\zeta_{\iota}\right)$ of $\nu_{\iota}$-measurable field of operators for $i \in \Lambda$ such that $\mathfrak{N}_{\iota}\left(\zeta_{\iota}\right)$ is the von Neumann algebra generated by the countable set $\left\{A_{i(\iota), \iota}\left(\zeta_{\iota}\right): i \in \Lambda\right\} \quad \nu_{\iota}$-almost everywhere on $Z_{\iota}$. Define $A_{0, \iota}=1_{\iota}$ and $A_{0, \iota}\left(\zeta_{\iota}\right)=1\left(\zeta_{\iota}\right)$. Denote $A_{i, \iota}=A_{i(\iota), \iota}$ and $A_{i, \iota}\left(\zeta_{\iota}\right)=A_{i(\iota), \iota}\left(\zeta_{\iota}\right)$ in the following. According to Lemma 4.3, if

$$
A_{i, \iota}=\int_{Z_{\iota}}^{\oplus} A_{i, \iota}\left(\zeta_{\iota}\right) d \nu_{\iota}\left(\zeta_{\iota}\right)
$$

for $\iota \in I$ and $i \in \Lambda$, then

$$
\otimes^{c} A_{i, \iota}=\int_{Z}^{\oplus} \otimes^{c(\zeta)} A_{i, \iota}\left(\zeta_{\iota}\right) d\left(\otimes \nu_{\iota}\right)(\zeta)
$$

for $\zeta=\left(\zeta_{\imath}\right)$, in which we denote by $A_{i}=\otimes^{c} A_{i, \iota}$ and also $A_{i}(\zeta)=\otimes^{c(\zeta)} A_{i, \iota}\left(\zeta_{\iota}\right)$. Since $\Lambda$ is countable set by (A1), $\otimes^{\mathrm{c}(\zeta)} \mathfrak{2}_{\imath}\left(\zeta_{\iota}\right)$ is generated by the countable set $\left\{A_{i}(\zeta): i \in \Lambda\right\} \otimes \nu_{\iota}$-almost everywhere on $Z$ and hence

$$
\zeta=\left(\zeta_{\iota}\right) \rightarrow \otimes^{c(\zeta) \mathfrak{A}_{\imath}\left(\zeta_{\iota}\right)}
$$

is a $\otimes \nu_{\iota}$-measurable field of von Neumann algebras. Since $\otimes \mathfrak{2} \mathfrak{A}_{\iota}$ contains $\left\{A_{i}: i \in \Lambda\right\}$, we have that

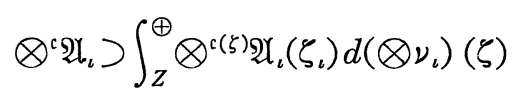

for $\zeta=\left(\zeta_{\iota}\right) \in Z$. According to (A3), we have

$$
\mathfrak{N}_{\iota}^{\prime}=\int_{Z_{\iota}}^{\oplus} \mathfrak{N}_{\iota}\left(\zeta_{\iota}\right)^{\prime} d \nu_{\iota}\left(\zeta_{\iota}\right)
$$

Since in the previous Lemmas 4.2 and 4.3, we have not use the assumption (A5), repeating the similar arguments as above, we can conclude that

$$
\left.\otimes^{\prime} \mathfrak{A}_{\imath}^{\prime}\right) \int_{Z}^{\oplus} \otimes^{\mathfrak{c}(\zeta)} \mathfrak{Q}_{\iota}\left(\zeta_{\iota}\right)^{\prime} d\left(\otimes \nu_{\imath}\right)(\zeta)
$$

Here we apply the results on the commutation theorem in an incomplete 
infinite tensor product $\left[3 ;\right.$ p. 94], then we have $\left(\otimes^{\mathfrak{c}} \mathfrak{U}_{\iota}\right)^{\prime}=\otimes^{\prime} \mathfrak{U}_{\iota}^{\prime}$. Again from (A1) and (A3), we get

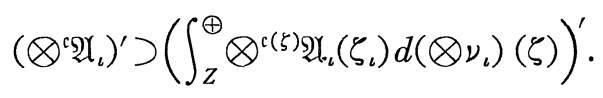

Lemma 4.5. Let $Y$ be a compact Hausdorff space, $\mu$ a positive Radon measure on $Y$ with $\|\mu\|=1, \zeta \rightarrow \mathfrak{S}(\zeta)$ a $\mu$-measurable field of Hilbert spaces on $Y, \zeta \rightarrow \mathfrak{A}(\zeta)$ a $\mu$-measurable field of von Neumann algebras on $\mathfrak{S}(\zeta)$ on $Y, \zeta \rightarrow \varphi_{\zeta}$ a field of normal traces on $\mathfrak{A}(\zeta)$ on $Y$,

$$
\mathfrak{L}=\int_{Y}^{\oplus} \mathfrak{S}(\zeta) d \mu(\zeta), \quad \mathfrak{A}=\int_{Y}^{\oplus} \mathfrak{A}(\zeta) d \mu(\zeta)
$$

and $\mathfrak{H}_{0}$ a weakly dense sub-*-algebra of $\mathfrak{A}$. Denote the decomposable operator $A$ in $\mathfrak{U}$ by

$$
A=\int_{Y}^{\oplus} A(\zeta) d \mu(\zeta)
$$

Then, if $\zeta \rightarrow \varphi_{\zeta}(A(\zeta))$ is $\mu$-measurable for every $A \in \mathfrak{A}_{0}$, then $\zeta \rightarrow \varphi_{\zeta}$ is $\mu$ measurable. Further, if there is a normal trace $\varphi$ on $\mathfrak{A}$ such that

$$
\varphi(A)=\int \varphi_{\zeta}(A(\zeta)) d \mu(\zeta)
$$

for every $A \in \mathfrak{A}_{0}$, then

$$
\varphi(A)=\int \varphi_{\zeta}(A(\zeta)) d \mu(\zeta)
$$

for every $A \in \mathfrak{2}$.

Proof. As $\mathfrak{N}$. is countably decomposable, if $A \in \mathfrak{A}$, then by the density theorem of Kaplansky, there is a sequence $\left\{A_{i}: i=1,2, \ldots\right\}$ in $\mathfrak{N}_{0}$ such that $A_{i}$ converges strongly to $A$ as $i$ tends $+\infty$ and $\left\|A_{i}\right\| \leqq\|A\|$. Since each $A_{i}$ is decomposable, say

$$
A_{i}=\int_{Y}^{\oplus} A_{i}(\zeta) d \mu(\zeta)
$$

there exists a subsequence $\left\{A_{i j}: j=1,2, \ldots\right\}$ satisfying that there is a $\mu$ null set $N$ such that $\left\|A_{i j}(\zeta)\right\| \leqq\|A\|$ and $A_{i j}(\zeta)$ converges strongly to $A(\zeta)$ as $j$ tends to $+\infty$ for $\zeta \in Z-N$. Hence there is $\mu$-null set $N^{\prime}$ with 
$N \subset N^{\prime}$ such that $\varphi_{\zeta}\left(A_{i j}(\zeta)\right)$ converges to $\varphi_{\zeta}(A(\zeta))$ for $\zeta \in Z-N^{\prime}$. This tells us that a sequence of $\mu$-measurable functions $\zeta \rightarrow \varphi_{\zeta}\left(A_{i j}(\zeta)\right)$ converges $\mu$-almost everywhere, boundedly to some function $\zeta \rightarrow \varphi_{\zeta}(A(\zeta))$, which is $\mu$-measurable. Hence by the bounded convergence theorem of Lebesgue

$$
\int_{Y} \varphi_{\zeta}\left(A_{i j}(\zeta)\right) d \mu(\zeta) \rightarrow \int_{Y} \varphi_{\zeta}(A(\zeta)) d \mu(\zeta)
$$

as $j$ tends to $+\infty$. On the other hand, $\varphi\left(A_{i j}\right)$ converges to $\varphi(A)$, hence the desired equality is obtained.

Q.E.D.

Lemma 4.6. Assume (A2), (A3), (A5), (A6) and $J \Subset I$. Then

(i) there is a $\otimes_{J} \nu_{\iota}$-measurable field $\zeta=\left(\zeta_{\iota}\right) \rightarrow\left(\otimes_{J} \psi\right)_{\zeta}$ of normal traces with $\left\|\left(\otimes_{J} \psi\right)_{\zeta}\right\|=1 \otimes_{J} \nu_{\imath}$-almost everywhere on $Z(J)$; and

(ii) the necessary and sufficient condition that $\varphi$ is a normal trace

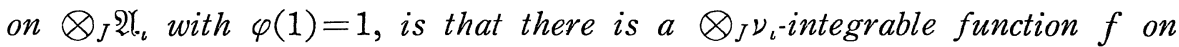
$Z(J)$ with $i f \|_{11}=1$ such that

$$
\varphi=\int_{Z(J)}^{\oplus} f(\zeta)(\underset{J}{\otimes} \psi)_{\zeta} d\left(\underset{J}{\otimes} \nu_{\iota}\right)(\zeta)
$$

Proof. By (A3), (A5) and (A6), there is a $\nu_{\iota}$-null set $N_{\iota}$ such that $\mathfrak{A}_{\iota}\left(\zeta_{\iota}\right)$ is a finite factor for $\zeta_{\iota} \in Z_{\iota}-N_{\iota}$ and for $\iota \in J$. Hence a decomposition obtained in Lemma 4.4,

$$
\underset{J}{\otimes} \mathscr{P}_{\cdot \iota}=\int_{Z(J)}^{\oplus} \underset{J}{\otimes \mathfrak{N}_{\iota}}\left(\zeta_{\iota}\right) d\left(\underset{J}{\otimes} \nu_{\iota}\right)(\zeta)
$$

is a factor decomposition. Since $\mathfrak{V}_{\iota}\left(\zeta_{\iota}\right)$ is a finite factor for $\zeta_{\iota} \in Z_{\iota}-N_{\iota}$, there is a canonical trace $\psi_{\zeta_{\text {c }}}$ on $\mathfrak{2}_{\iota}\left(\zeta_{\iota}\right)$ and hence there is a canonical trace $\left(\otimes_{J} \psi\right)_{\zeta}=\otimes_{J} \psi_{\zeta_{\iota}}$ on a finite factor $\otimes_{J} \mathfrak{2}_{\iota}\left(\zeta_{\iota}\right)$ for $\zeta=\left(\zeta_{\iota}\right) \in Z_{0}=\Pi_{J}\left(Z_{\iota}-N_{\iota}\right)$ and $\left(\otimes_{J} \nu_{\iota}\right)\left(Z_{0}\right)=1$. Define $\left(\otimes_{J} \psi\right)_{\zeta}=0$ for $\zeta \in Z(J)-Z_{0}$. Then $\zeta \rightarrow\left(\otimes_{J} \psi\right)_{\zeta}$ is a field of normal traces on $Z(J)$. On the other hand, $\varphi$ is a normal trace on $\otimes_{J} \mathfrak{A}_{\iota}$ with $\varphi(1)=1$, then by (A3) there exists a $\otimes_{J} \nu_{\iota}$-measurable field $\zeta \rightarrow \varphi_{\zeta}$ of normal traces on $\otimes_{J} \mathfrak{A}_{\imath}\left(\zeta_{\iota}\right)$ such that

$$
\varphi=\int_{Z(J)}^{\oplus} \varphi_{\zeta} d\left(\underset{J}{\left.\otimes \nu_{\iota}\right)(\zeta)}\right.
$$

and $\varphi_{\zeta}$ is a finite normal trace for $\zeta \in Z_{0}-N$ with $\left(\otimes_{J} \nu_{\iota}\right)(N)=0$. Since 
$\otimes_{J} \mathfrak{R}_{\iota}\left(\zeta_{\iota}\right)$ is a finite factor for $\zeta \in Z_{0}$, we have $\varphi_{\zeta}=\varphi_{\zeta}(1)\left(\otimes_{J} \psi\right)_{\zeta}$ for $\zeta \in Z_{0}-N$. Define $f(\zeta)=\varphi_{\zeta}(1)$. Then $f(\zeta)$ is $\otimes_{J} \nu_{\iota}$-measurable. Since $\otimes_{J} \mathfrak{U}_{\iota}$ is countably decomposable, we may choose a finite faithful normal trace as $\varphi$ and hence $\zeta \rightarrow \varphi_{\zeta}$ is a $\otimes_{J} \nu_{\iota}$-measurable field of finite faithful normal traces. Therefore $\zeta \rightarrow \varphi_{\zeta}(1)$ is non zero $\otimes \nu_{\iota}$-almost everywhere. Define

$$
g(\zeta)=\left\{\begin{array}{cc}
\varphi_{\zeta}(1)^{-1} & \text { if } \varphi_{\zeta}(1) \neq 0 \text { and } \zeta \in Z_{0}-N \\
0 & \text { otherwise. }
\end{array}\right.
$$

Then $g(\zeta)$ is $\otimes_{J} \nu_{\imath}$-measurable. Thus $\zeta \rightarrow g(\zeta) \varphi_{\zeta}$ is $\otimes_{J} \nu_{\iota}$-measurable and therefore $\zeta \rightarrow\left(\otimes_{J} \psi\right)_{\zeta}$ is $\otimes_{J} \nu_{\imath}$-measurable.

Q.E.D.

Remark 4.2. Denote by $\nu$ the measure defined by $d \nu(\zeta)=f(\zeta) d\left(\otimes_{J} \nu_{\iota}\right)(\zeta)$. Then a normal trace $\varphi$ with $\|\varphi\|=1$ and a probability measure $\nu$ have a one to one correspondence. Moreover, the field $\zeta=\left(\zeta_{\iota}\right) \rightarrow\left(\otimes_{J} \psi\right)_{\zeta}$ is $\nu$ measurable for every $\nu$ which is associated with $\varphi$ on $\otimes_{J} \mathfrak{R}$.

If $\{\nu(J): J \ll I\}$ is a projective system on $Z=\Pi Z_{\iota}$ of probability measures $\nu(J)$ on $Z(J)=\prod_{J} Z_{\iota}$ then it is well known that there exists a unique probability measure $\nu$ on $Z$ whose projection onto $Z(J)$ is $\nu(J)$ for $J \ll I,[7]$.

Lemma 4.7. Assume (A2), (A3), (A5) and (A6). Given a normal trace $\varphi$ on $\otimes^{c} \mathfrak{R}_{\imath}$ with $\varphi(1)=1$. If $\nu(J)$ is a probability measure on $Z(J)$ corresponding to the projection $\varphi(J)$ of $\varphi$ to $\otimes_{J} \mathfrak{R l}_{\text {. }}$ as Remark 4.2 such that

$$
\varphi(J)=\int_{Z(J)}^{\oplus}\left(\bigotimes_{J} \psi\right)_{\zeta} d \nu(J)(\zeta)
$$

for $\zeta=\left(\zeta_{\iota}\right) \in Z(J)$, then $\{\nu(J): J \ll I\}$ is a projective system of probability measures and so there is a probability measure $\nu$ on $Z$ such that $\nu=\lim _{\longleftarrow} \nu(J)$.

Proof. Suppose $J \subset K \ll I$. If $A \in \bigotimes_{J} \mathfrak{U}_{\iota}$ and $1 \in \bigotimes_{K-J} \mathfrak{H}_{\iota}$ such that

$$
A=\int_{Z(J)}^{\oplus} A(\zeta) d \nu(J)(\zeta) \text { and } \quad 1=\int_{Z(K-J)}^{\oplus} 1(\eta) d \nu(K-J)(\eta)
$$

then by Lemma 4.3 


$$
A \otimes 1=\int_{Z(K)}^{\oplus} A(\zeta) \otimes 1(\eta) d(\nu(J) \otimes \nu(K-J))(\zeta, \eta) .
$$

By the definition of $\nu(K)$,

$$
\varphi(K)(A \otimes 1)=\int_{Z(K)}\left(\otimes_{J} \psi\right)_{\zeta}(A(\zeta)) 1(\eta) d \nu(K)(\zeta, \eta),
$$

where $\zeta=\left(\zeta_{\imath}\right) \in Z(J)$ and $\eta=\left(\eta_{\imath}\right) \in Z(K-J)$. Further

$$
\varphi(J)(A)=\int_{Z(J)}\left(\bigotimes_{J} \psi\right)_{\zeta}(A(\zeta)) d \nu(J)(\zeta)
$$

Combining the last two equalities, we find that $\nu(J)$ is the projection of $\nu(K)$ by the arbitrariness of $A \in \otimes_{J} \mathfrak{A}_{\iota}$.

Q.E.D.

Lemma 4.8. Assume (A2), (A3), (A4), (A5) and (A6). Then there

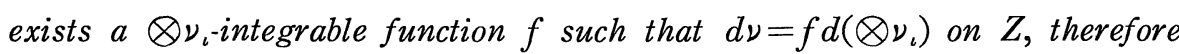
$\nu$ is absolutely continuous with respect to $\otimes \nu_{\iota}$.

Proof. According to Remark 4.2 there is a $\otimes_{J} \nu_{\iota}$-integrable function $f(J)$ such that $d \nu(J)=f(J) d\left(\otimes_{J} \nu_{\iota}\right)$ on $Z(J)$ for $J \ll I$. We define

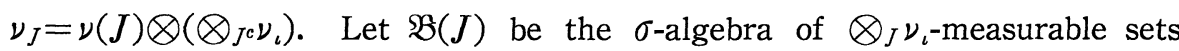
and $\mathfrak{B}_{J}$ the set of $B(J) \times Z\left(J^{c}\right)$ for every $B(J) \in \mathfrak{B}(J)$. Then $\left\{\mathfrak{B}_{J}: J \Subset I\right\}$ is a monotone increasing net of $\sigma$-algebra which is contained in the $\sigma$ algebra $\mathfrak{B}$ of $\otimes \nu_{\imath}$-measurable sets, when $J$ tends to $I$ by the order of set inclusion. Denote by $E\left\{\cdot \mid \mathfrak{B}_{J}\right\}$ the conditional expectation with respect to $\mathfrak{B}_{J}$ for $J \ll I$. Define $f_{J}=f(J) \otimes 1\left(J^{c}\right)$, where $1\left(J^{c}\right)$ is a constant 1 function on $Z\left(J^{c}\right)$. Then $\left\{f_{J}: J \ll I\right\}$ is a martingale with respect to $\otimes \nu_{\iota}$, because $E\left\{f_{K} \mid \mathfrak{B}_{J}\right\}=f_{J}$ except on $\otimes \nu_{\iota}$-null set whenever $J \subset K \Subset I$. It follows from the convergence theorem of martingale [10] that there is a $\otimes \nu_{\imath}$-integrable function $f$ on $Z$ such that $f_{J}$ converges to $f$ in the $L^{1}$-mean and $\|f\|_{1}=1$ due to $\left\|f_{J}\right\|_{1}=1$. Here we denote $d \mu=f d\left(\otimes \nu_{\iota}\right)$. Then $\mu$ is a Radon measure on $Z$ with $\|\mu\|=1$. Let $\mathfrak{P}$ be the union of all sets of functions $g=g(J) \otimes 1\left(J^{c}\right)$ for $g(J) \in C(Z(J))$ when $J$ runs over $J \Subset I$. Then, by means of the Stone-Weierstrass theorem, $\mathfrak{F}$ is uniformly dense in $C(Z)$. From this we know that $d \mu=f d\left(\otimes \nu_{\imath}\right)$ and $\nu=\lim \nu(J)$ coincide on $\mathfrak{P}$ and hence $\mu=\nu$ on $C(Z)$ Q.E.D.

It is immediate from this lemma that $\otimes \nu_{\imath}$-measurability implies 
$\nu$-measurability.

Let $Y$ be a compact Hausdorff space, $\mu$ a positive Radon measure on $Y$ whose carrier is $Y, \zeta \rightarrow \mathfrak{S}(\zeta)$ a $\mu$-measurable field of non null Hilbert spaces on $Y$,

$$
\mathfrak{S}=\int_{Y}^{\oplus} \mathfrak{S}(\zeta) d \mu(\zeta), \quad \mathfrak{U}=\int_{Y}^{\oplus} \mathfrak{\mathscr { U }}(\zeta) d \mu(\zeta)
$$

a finite decomposable von Neumann algebra on $\mathfrak{S}$ with the coupling operator $C$ and 3 the algebra of diagonalizable operators identified with $L^{\infty}(Y, \mu)$. Suppose that the center of $\mathscr{A}$ is 3 and $Y$ satisfies the second axiom of countability. Then it is known that for any $x$ in $\mathfrak{S}$ with

$$
x=\int_{Y}^{\oplus} x(\zeta) d \mu(\zeta)
$$

we have

$$
E\left(\mathfrak{U}^{\prime}, x\right)=\int_{Y}^{\oplus} E\left(\mathfrak{R}(\zeta)^{\prime}, x(\zeta)\right) d \mu(\zeta)
$$

and

$$
E(\mathfrak{A}, x)=\int_{Y}^{\oplus} E(\mathfrak{R}(\zeta), x(\zeta)) d \mu(\zeta)
$$

Denote the canonical $\sharp$-mapping on $\mathfrak{A}$ to 3 by $\Phi$. Let $\zeta \rightarrow \varphi_{\zeta}$ be a $\mu$ measurable field of canonical normal traces on $2(\zeta)$ on $Y$.

Since $\mathfrak{Q}$ is finite and 3 is the center of $\mathfrak{N}$, there is a $\mu$-null set $N$ such that $\mathfrak{A}(\zeta)$ is a finite factor for each $\zeta \in Y-N$. Hence the canonical 4-mapping $\Phi_{\zeta}$ on $\mathfrak{N}(\zeta)$ is a scalar valued function with $\varphi_{\zeta}\left(E\left(\mathfrak{H}(\zeta)^{\prime}, x(\zeta)\right)\right)$ $=\varphi_{\zeta}\left(\Phi_{\zeta}\left(E\left(\mathfrak{H}(\zeta)^{\prime}, x(\zeta)\right)\right)\right)$ for $\zeta \in Y-N$ and hence $\Phi_{\zeta}\left(E\left(\mathfrak{A}(\zeta)^{\prime}, x(\zeta)\right)\right)$ $=\varphi_{\zeta}\left(E\left(\mathfrak{R}_{(}(\zeta)^{\prime}, x(\zeta)\right)\right)$ for $\zeta \in Y-N$. Thus we have

$$
\Phi\left(E\left(\mathfrak{A}^{\prime}, x\right)\right)=\int_{Y}^{\oplus} \Phi_{\zeta}\left(E\left(\mathfrak{U}(\zeta)^{\prime}, x(\zeta)\right)\right) d \mu(\zeta) .
$$

Similarly if $\mathfrak{Q}^{\prime}$ is finite, then there is a $\mu$-null set $N^{\prime}$ such that $\mathfrak{N}(\zeta)^{\prime}$ is finite $\zeta \in Y-N^{\prime}$. Denoting the canonical 4 -mappings on $\mathfrak{N}^{\prime} \cdot$ and $\mathscr{N}(\zeta)^{\prime}$ by $\Phi^{\prime}$ and $\Phi_{\zeta}^{\prime}$ for $\zeta \in Y-N^{\prime}$, we have

$$
\Phi^{\prime}(E(\mathfrak{A}, x))=\int_{Y}^{\oplus} \Phi_{\zeta}^{\prime}(E(\mathfrak{Y}(\zeta), x(\zeta))) d \mu(\zeta) .
$$


Since 3 is identified with $L^{\infty}(Y, \mu), C$ is an element $\hat{Z}^{+}$, that is, a non negative $\mu$-measurable function on $Y$, which may be written formally in the form;

$$
C=\int_{Y}^{\oplus} C(\zeta) d \mu(\zeta)
$$

Consequently the function $\zeta \rightarrow \Phi_{\zeta}\left(E\left(\mathscr{R}(\zeta)^{\prime}, x(\zeta)\right)\right)$ coincides with the function $\zeta \rightarrow C(\zeta) \Phi_{\zeta}^{\prime}(E(\Re(\zeta), x(\zeta)))$ except a $\mu$-null set $N_{0}$ with $N \cup N^{\prime} \subset N_{0}$, that is, $C(\zeta)$ is the coupling constant of $\mathfrak{A}(\zeta)$ for each $\zeta \in Y-N_{0}$, which is written in the form;

$$
\int_{Y}^{\oplus} \Phi_{\zeta}\left(E\left(\mathfrak{A}(\zeta)^{\prime}, x(\zeta)\right)\right) d \mu(\zeta)=\int_{Y}^{\oplus} C(\zeta) \Phi_{\zeta}^{\prime}(E(\mathfrak{A} .(\zeta), x(\zeta))) d \mu(\zeta) .
$$

When $\mathfrak{X}^{\prime}$ is not finite, it is decomposed into a finite $\mathfrak{U}_{G}^{\prime}$ and a properly infinite $\mathfrak{A}_{1-G}^{\prime}$ by the projection $G$ in $\mathfrak{A}^{\prime} \cap \mathfrak{A}^{\prime} . \quad C_{G}$, the coupling operator of $\mathfrak{2}_{G}$ is similarly treated as above on $G \mathfrak{S}$. On the other hand $C_{1-G}=+\infty$ on $(1-G) \mathfrak{S}$ and

$$
\mathfrak{A}_{1-G}^{\prime}=\int_{Y}^{\oplus} \mathfrak{Y}_{(1-G)(\zeta)}^{\prime}(\zeta) d \mu(\zeta)
$$

where

$$
1-G=\int_{Y}^{\oplus}(1-G)(\zeta) d \mu(\zeta)
$$

Since $\mathfrak{A}_{1-G}$ is properly infinite, there is a $\mu$-null set $N_{1}$ such that $\mathfrak{U}_{(1-G)(\zeta)}^{\prime}(\zeta)$ is a properly infinite factor for $\zeta \in Y-N_{1}$. Hence we can define the coupling operator of $\mathfrak{2}(\zeta)$ by $C(\zeta)=+\infty$ for $\zeta \in Y-N_{1}$. Consequently, $C(\zeta)$ is the coupling constant $\mu$-almost everywhere on $Y$.

By virtue of these considerations we have the following Lemma.

Lemma 4.9. Assume (A1), (A2), (A3), (A4), (A6) and (A7). If $\otimes^{c} \mathfrak{N}_{\text {. }}$ has a non trivial finite part for $\mathrm{c} \in \Gamma$, then there is a normal trace $\psi_{\zeta}^{c}$ on $\otimes^{c(\zeta)} \mathfrak{2}_{\iota}\left(\zeta_{\iota}\right)$ for $\otimes \nu_{\iota}$-almost everywhere on $Z$ such that for every probability measure $\nu$ associated with a normal trace $\varphi$ on $\otimes^{c} \mathfrak{A}_{\iota}$ with $\|\varphi\|=1, \psi_{\zeta}^{c}$ is a canonical trace on $\otimes^{\mathrm{c}(\zeta)} \mathfrak{M}_{\iota_{2}}\left(\zeta_{\iota}\right)$ for $\nu$-almost everywhere on $Z$. 
Proof. By (A3), (A5) and (A6), there is a $\nu_{\iota}$-null set $N_{\iota}$ such that $\mathfrak{A}_{\iota}\left(\zeta_{\iota}\right)$ is a finite factor for each $\zeta_{\iota} \in Z_{\iota}-N_{\iota}$. Hence there is a canonical trace $\psi_{\zeta_{\iota}}$ on $\mathfrak{N}_{\iota}\left(\zeta_{\iota}\right)$ for $\zeta_{\iota} \in Z_{\iota}-N_{\iota}$. According to the above arguments, there is a $\nu_{\iota}$-null set $N_{\iota}^{\prime}$ with $N_{\iota} \subset N_{\iota}^{\prime}$ such that $C_{\iota}\left(\zeta_{\iota}\right)$ is the coupling constant of $\mathfrak{N}_{\iota}\left(\zeta_{\iota}\right)$ for $\zeta_{\iota} \in Z_{\iota}-N_{\iota}^{\prime}$. Since $\left(1-C_{\iota}\right)^{+}\left(\zeta_{\iota}\right)=\max \left\{1-C_{\iota}\left(\zeta_{\iota}\right), 0\right\}$ $\nu_{\iota}$-almost everywhere and (A7), there is a $\nu_{\imath}$-null set $N_{\iota}^{\prime \prime}$ with $N_{\iota}^{\prime} \subset N_{\iota}^{\prime \prime}$ such that $\max \left\{1-C_{\iota}\left(\zeta_{\iota}\right), 0\right\} \leqq\left\|\left(1-C_{\iota}\right)^{+}\right\|$for $\zeta_{\iota} \in Z_{\iota}-N_{\iota}^{\prime \prime}$. Therefore we have

$$
\sum \max \left\{1-C_{\iota}\left(\zeta_{1}\right), 0\right\} \leqq \sum\left\|\left(1-C_{\imath}\right)^{+}\right\|<+\infty
$$

for any $\zeta=\left(\zeta_{\iota}\right)$ in $Z_{0}=\Pi\left(Z_{\iota}-N_{\iota}^{\prime \prime}\right)$, and hence a normal trace $\psi_{\zeta}=\otimes \psi_{\zeta_{\iota}}$ on $\otimes \mathfrak{R}_{\iota}\left(\zeta_{\iota}\right)$ can be defined for $\zeta \in Z_{0}$ and $\left(\otimes \nu_{\iota}\right)\left(Z_{0}\right)=1$.

Let $\left(e_{\iota}\right) \in \mathfrak{c}$,

$$
e_{\iota}=\int_{Z_{\iota}}^{\oplus} e_{\iota}\left(\zeta_{\iota}\right) d \nu_{\iota}\left(\zeta_{\iota}\right)
$$

$\left\|e_{\iota}\left(\zeta_{\iota}\right)\right\|=1$ and $\left(e_{\iota}\left(\zeta_{\iota}\right)\right) \in \mathfrak{c}(\zeta)$. Let $x_{\iota}\left(\zeta_{\iota}\right)$ be a characteristic vector of $\psi_{\zeta_{\iota}}$ and $\mathfrak{c}(\zeta)^{\prime}$ a characteristic class with $\left(x_{\iota}\left(\zeta_{\iota}\right)\right) \in \mathfrak{c}(\zeta)^{\prime}$. Then by Corollary 3.4 , the carrier of $\psi_{\zeta}$ is majorated by $P\left(\mathfrak{c}(\zeta)^{\prime}\right)$. According to Theorem 3.1 , for any $\varepsilon>0$ there exists $J \ll I$ such that

$$
\left\|\varphi(K)-\omega_{e(K)}\right\|<\varepsilon
$$

for every $K \ll J^{c}$. Denote $\mathfrak{A}(K)=\bigotimes_{K} \mathfrak{A}_{\iota}$ and $\mathfrak{A}(K)(\zeta)=\bigotimes_{K} \mathfrak{N}_{\iota}\left(\zeta_{\iota}\right)$ for any $K \ll I$. Since by (A3), Remark 4.1 and Lemma 4.4

$$
E\left(\mathfrak{A}(K)^{\prime}, e(K)\right)=\int_{Z(K)}^{\oplus} E\left(\mathfrak{A}(K)(\zeta)^{\prime}, e(K)(\zeta)\right) d \nu(K)(\zeta)
$$

we have $\left|\varphi(K)\left(E\left(\mathfrak{Y}(K)^{\prime}, e(K)\right)\right)-1\right|<\varepsilon$ and

$$
\begin{aligned}
& \varphi(K)\left(E\left(\mathfrak{X}(K)^{\prime}, e(K)\right)\right) \\
& \quad=\int_{Z(K)}\left(\bigotimes_{K} \psi\right)_{\zeta}\left(E\left(\mathfrak{H}(K)(\zeta)^{\prime}, e(K)(\zeta)\right)\right) d \nu(K)(\zeta),
\end{aligned}
$$

and hence there is a $\nu(K)$-measurable set $M C Z(K)$ such that $\nu(K)(M)<\sqrt{\varepsilon}$ and

$$
\left|\left(\bigotimes_{K} \psi\right)_{\zeta}\left(E\left(\mathfrak{Y} .(K)(\zeta)^{\prime}, e(K)(\zeta)\right)\right)-1\right|<\sqrt{\varepsilon}
$$


for $\zeta \in Z(K)-M$. For any $\varepsilon>0$, put $\varepsilon_{i}=\varepsilon^{2} / 4^{i}$ for $i=1,2, \ldots$ Then for these $\varepsilon_{i}$ there is $J_{i} \ll I$ such that $J_{i} \uparrow I$ and

$$
\left\|\varphi\left(K_{i}\right)-\omega_{e\left(K_{i}\right)}\right\|<\varepsilon_{i},
$$

where $K_{i}=J_{i+1}-J_{i}$. Thus for every $i$ there is a $\nu\left(K_{i}\right)$-measurable $M_{i}$ such that $\nu\left(K_{i}\right)\left(M_{i}\right)<\sqrt{\varepsilon_{i}}$ and

$$
\left|\left(\bigotimes_{K_{i}} \psi\right)_{\zeta}\left(E\left(\mathfrak{Y}\left(K_{i}\right)(\zeta)^{\prime}, e\left(K_{i}\right)(\zeta)\right)\right)-1\right|<\sqrt{\varepsilon_{i}}
$$

for $\zeta \in Z\left(K_{i}\right)_{0}-M_{i}$, where $Z\left(K_{i}\right)_{0}=\Pi_{K_{\imath}}\left(Z_{\iota}-N_{\iota}^{\prime \prime}\right)$. Denote $J=J_{1}$ and $M\left(J^{c}\right)=\bigcup_{i=1}^{\infty} M_{i} \times Z\left(J^{c}-K_{i}\right)$. Then $M\left(J^{c}\right)$ is $\otimes_{J^{c} \nu_{\iota}}$-measurable,

$$
\nu\left(J^{c}\right)\left(M\left(J^{c}\right)\right) \leqq \sum \nu\left(J^{c}\right)\left(M_{i} \times Z\left(J^{c}-K_{i}\right)\right)<\varepsilon
$$

and

$$
\left|\left(\bigotimes_{J^{c}} \psi\right)_{\zeta}\left(E\left(\Re\left(J^{c}\right)(\zeta)^{\prime}, e\left(J^{c}\right)(\zeta)\right)\right)-1\right|<\varepsilon
$$

for $\zeta \in Z\left(J^{c}\right)_{0}-M\left(J^{c}\right)$, where $Z\left(J^{c}\right)_{0}=\Pi_{J^{c}}\left(Z_{\iota}-N_{\iota}^{\prime \prime}\right)$. Consequently by Corollary 4.4 in $[6]$ we have $\otimes e_{\iota}\left(\zeta_{\iota}\right)=P\left(\mathfrak{c}(\zeta)^{\prime}\right)\left(\otimes e_{\iota}\left(\zeta_{\iota}\right)\right)$, that is, $P(\mathfrak{c}(\zeta))=P\left(\mathfrak{c}(\zeta)^{\prime}\right)$ for $\zeta \in Z_{0}-M$, where $M=\bigcup_{i=1}^{\infty} M_{i} \times Z\left(K_{i}^{c}\right)$ is $\nu$-measurable. On the other hand, since $\otimes^{c(\zeta)} \mathscr{U}_{\iota}\left(\zeta_{\iota}\right)$ and $\otimes^{(c(\zeta))} \mathfrak{A}_{\iota}\left(\zeta_{\iota}\right)$ is isomorphic, we may consider $\phi_{\zeta}$ for $\zeta \in Z_{0}$ to be a normal trace on $\otimes^{c(\zeta)} \mathscr{N}_{c}\left(\zeta_{\iota}\right)$ by the restriction, which we denote by $\psi_{\zeta}^{c}$. Owing to the arbitrariness of $\varepsilon$ we may select a monotone decreasing sequence of $\nu$-measurable sets $M \subset Z_{0}$ when $\varepsilon$ tends to 0 . Denote the limit set by $M_{0}$. Then $\nu\left(M_{0}\right)=0$ and $\psi_{\zeta}^{c}$ is a canonical trace on $\otimes^{c(\zeta)} \mathfrak{U}_{\iota}\left(\zeta_{\iota}\right)$ for $\zeta \in Z_{0}-M_{0}$.

Q.E.D.

Lemma 4.10. Assume (A1), (A2), (A3), (A4), (A5), (A6) and (A7). If $\varphi$ is a normal trace on $\otimes^{c} \mathfrak{U}_{\llcorner}$with $\|\varphi\|=1$ and $\nu$ its associated probability measure on $Z$, then the field $\zeta \rightarrow \psi_{\zeta}^{c}$ of normal traces in the last lemma is $\nu$-measurable and

$$
\varphi=\int_{Z}^{\oplus} \psi_{\zeta}^{\mathrm{c}} d \nu(\zeta)
$$

Proof. If $A \in \otimes_{J} \mathfrak{A}$, for $J \ll I$ such that 


$$
A=\int_{Z(J)}^{\oplus} A(\zeta) d\left(\underset{J}{\otimes} \nu_{\iota}\right)(\zeta)
$$

then, by Lemma $4.6, \zeta \rightarrow\left(\otimes_{J} \psi\right)_{\zeta}(A(\zeta))$ is $\nu(J)$-measurable. Let $1\left(\zeta\left(J^{c}\right)\right)$ be the identities in $\otimes_{J^{c}}^{c(\zeta)} \mathfrak{A}_{\iota}\left(\zeta_{\iota}\right)$, where $\zeta(K)=\left(\zeta_{\iota}: \iota \in K\right)$ for any $K \subset I$. Denote $A_{J}(\zeta)=A(\zeta(J)) \otimes 1\left(\zeta\left(J^{c}\right)\right)$. Then the field

$$
\zeta \rightarrow \phi_{\zeta}^{c}\left(A_{J}(\zeta)\right)=\left(\bigotimes_{J} \psi\right)_{\zeta(J)}(A(\zeta(J))) 1\left(\zeta\left(J^{c}\right)\right)
$$

is $\nu$-measurable on $Z$. Thus, by Lemmas 4.5 and 4.8 , we find that $\zeta \rightarrow \psi_{\zeta}^{\text {c }}$ is $\nu$-measurable and there is a normal trace $\psi$ on $\otimes^{c} \mathfrak{A}_{\iota}$ such that

$$
\psi=\int_{Z}^{\oplus} \psi_{\zeta}^{c} d \nu(\zeta)
$$

Since $\phi$ is a normal trace on $\otimes^{c} \mathscr{N}_{\iota}$ with $\phi(1)=1$, and $\varphi(J)=\psi(J)$ for all $J \Subset I$, it follows Theorem 3.1 that $\varphi=\psi$.

If $P_{\mathfrak{c}}$ and $P_{\mathrm{c}^{\prime}}$ for $\mathrm{c}, \mathrm{c}^{\prime} \in \Gamma$ have the same central carrier projection, then $\otimes^{c} \mathfrak{A}_{.}$and $\otimes^{c^{\prime}} \mathfrak{N}_{\iota}$ are isomorphic. But if their carriers are different, then $\otimes^{c} \mathscr{N}_{\iota}$ and $\otimes^{c^{\prime}} \mathfrak{U}_{\iota}$ are not always isomorphic. This situation becomes much clear from the following lemma.

Lemma 4.11. Assume (A2), (A3), (A4), (A5), (A6) and (A7). Given normal traces $\varphi$ on $\otimes^{c} \mathfrak{A}_{\iota}$ and $\psi$ on $\otimes^{c^{\prime}} \mathfrak{U}_{\iota}$ with $\varphi(1)=\psi(1)=1$, which are associated with probability measures $\nu$ and $\mu$ as in Lemma 4.7 respectively. If $P(\mathrm{c}) P\left(\mathrm{c}^{\prime}\right)=0$, then $\nu$ and $\mu$ are singular.

Proof. Let $E$ and $F$ be the carriers of $\varphi$ and $\psi$ such that

$$
E=\int_{Z}^{\oplus} E(\zeta) d\left(\otimes \nu_{\iota}\right)(\zeta) \text { and } F=\int_{Z}^{\oplus} F(\zeta) d\left(\otimes \nu_{\iota}\right)(\zeta)
$$

It follows from $E F=0$ that $E(\zeta) F(\zeta)=0 \otimes \nu_{\imath}$-almost everywhere on $Z$. Denote by $A$ and $B$ the sets of $\zeta \in Z$ with $E(\zeta)=1(\zeta)$ and $F(\zeta)=1(\zeta)$ respectively. Then $A$ and $B$ are $\otimes \nu_{\iota}$-measurable and $\left(\otimes \nu_{\iota}\right)(A \cap B)=0$. According to the last lemma

$$
1=\varphi(E)=\int_{Z} \psi_{\zeta}^{\mathrm{c}}(E(\zeta)) d \nu(\zeta)
$$


hence the carrier of $\nu$ is contained in $A \nu$-almost everywhere. Denote by $A^{\prime}$ the carrier of $\nu$ and define

$$
E^{\prime}=\int_{Z}^{\oplus} \chi_{A^{\prime}}(\zeta) d\left(\otimes v_{\iota}\right)(\zeta)
$$

Then we have

$$
\varphi\left(E^{\prime}\right)=\int_{Z} \psi_{\zeta}^{\mathrm{c}}\left(\chi_{A^{\prime}}(\zeta)\right) d \nu(\zeta)=1
$$

and therefore $E \leqq E^{\prime}$, that is, $A$ is $\nu$-almost everywhere contained in $A^{\prime}$. Hence by Lemma $4.8, A \ominus A^{\prime}$ is a $\nu$-null set and similarly $B \ominus B^{\prime}$ is a $\mu$-null set, where $B^{\prime}$ is the carrier of $\mu$. Since $\left(\otimes \nu_{\iota}\right)(A \cap B)=0$ and Lemma 4.8 , we have $\nu(A)=1$ and $\mu(A)=0$, moreover $\nu(B-A)=0$ and $\mu(B-A)=1$.

Q.E.D.

Lemma 4.12. Assume (A1), (A2), (A3), (A4), (A5), (A6) and (A7). Then there is a one and only one central projection $P(\mathfrak{c})$ in the set of all $P\left(\mathfrak{c}^{\prime}\right)$ 's for $\mathfrak{c}^{\prime} \in \Gamma$ satisfying that

(i) $\otimes^{(\mathfrak{c})} \mathfrak{A}_{.}$is finite;

(ii) if

$$
\otimes^{c} \mathfrak{R}_{\iota}=\int_{Z}^{\oplus} \otimes^{c(\zeta)} \mathfrak{N}_{\imath}\left(\zeta_{\iota}\right) d\left(\otimes \nu_{\iota}\right)(\zeta)
$$

then there exists a $\otimes v_{\iota}$-measurable field $\zeta \rightarrow \psi_{\zeta}^{c}$ of normal traces on

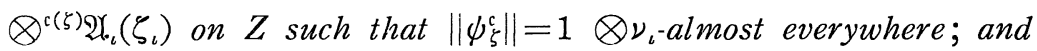

(iii) if $\varphi$ is a normal trace on $\otimes\left(\mathfrak{U}_{\iota}\right.$ with $\varphi(1)=1$ and $\nu$ a probability measure on $Z$ associated with $\varphi$, then $\otimes \nu_{\iota}$-measurable field $\zeta \rightarrow \phi_{\zeta}^{\mathrm{c}}$ in the above is $\nu$-measurable.

Proof. (i) By (A4), $\mathfrak{U}_{\iota}$ is countably decomposable for each $\iota \in I$. Hence there is a faithful normal trace $\varphi_{\iota}$ on $\mathfrak{U}_{\text {。 }}$ such that

$$
\varphi_{\iota}=\int_{Z_{\imath}}^{\oplus} \varphi_{\zeta_{\iota}} d \nu_{\iota}\left(\zeta_{\iota}\right)
$$

with $\left\|\varphi_{\zeta_{\iota}}\right\|=1 \quad \nu_{\iota}$-almost everywhere on $Z_{\iota}$. Since $\sum \varphi_{\iota}\left(\left(1-C_{\iota}\right)^{+}\right)$ $\leqq \sum\left\|\left(1-C_{\imath}\right)^{+}\right\|<+\infty$, we have a normal trace $\otimes \varphi_{\iota}$ on $\otimes 2 \mathrm{R}_{\iota}$ by Theorem 4.2 in $[6]$. Then there is a central $P(\mathfrak{c})$ such that the carrier of $\otimes \varphi$ ، 
is $P(\mathrm{c})$ by Corollary 4.2 in $[6]$. If there is another $P\left(c^{\prime}\right)$ which satisfies that a non trivial part of $\otimes^{\left(\mathfrak{c}^{\prime}\right)} \mathfrak{U}_{\iota}$ is finite, then a probability measure associated with a non zero finite normal trace on $\otimes^{c^{\prime}} \mathfrak{A}_{\iota}$ exists and is absolutely continuous with respect to $\otimes \nu$, by Lemma 4.8 , but it is singular to $\otimes \nu$, by Lemma 4.11 , which is a contradiction. Thus we obtain the uniqueness of $P(\mathrm{c})$.

(ii) Define a finite normal trace on $\mathfrak{2}_{.}$, by

$$
\varphi_{\iota}=\int_{Z_{\iota}}^{\oplus} \psi_{\zeta_{\iota}} d \nu_{\iota}\left(\zeta_{\iota}\right)
$$

Then $\varphi_{\iota}$ is faithful and $\varphi_{\iota}(1)=1$. Applying the same arguments as Lemma 4.8 , we can associate $\otimes \varphi_{\iota}$ with $\otimes \nu_{\imath}$. Then by Lemma 4.9 we have a $\otimes \nu_{\iota}$-measurable field $\zeta \rightarrow \psi_{\zeta}^{c}$ of normal traces on $\otimes^{c(\zeta)} \mathfrak{A}_{\iota}\left(\zeta_{\iota}\right)$ on $Z$ such that $\left\|\psi_{\xi}^{c}\right\|=1 \otimes \nu_{\iota}$-almost everywhere.

(iii) It is immediate from Lemma 4.1 . Q.E.D.

The main result is summarized already as Theorem in Section 1.

Acknowledgement. The author is indebted to Professor Araki for his many helpful discussions which have greatly contributed to improvements of this paper, to Dr. Kubo for his valuable discussions and to Professor Umegaki for his encouragement.

\section{References}

[1] Araki, H., A lattice of von Neumann algebras associated with the quantum theory of a free Bose field, J. Math. Phys. 4 (1963), 1343-1362.

[2] Araki, H. and E. J. Woods, Complete Boolean algebras of type I factors, Publ. PIMS, Kyoto Univ. 2 (1966), 157-242.

[3] Araki, H. and E. J. Woods, A classification of factors, Publ. RIMS, Kyoto Univ. 4 (1968), 51-130.

[4] Dixmier, J., Les algèbres d'opérateurs dans l'espace hilbertien, Gauthier-Villars, Paris, 1957.

[5] Dye, H. A., The Radon-Nikodým theorem for finite rings of operators, Trans. Amer. Math. Soc. 72 (1952), 243-280.

[6] Nakagami, Y., Infinite tensor products of von Neumann algebras, Kōdai Math. Sem. Rep., to appear.

[7] Schwartz, L., Les measures de Radon dans les espace topologiques arbitraires, Cours de 3d cycle 1964-1965, Inst. H. Poincaré, Paris.

[8 ] Segal, I. E., Decomposition of operator algebras I, and II, Mem. Amer. Math. Soc. 9 (1951), 1-67, and 1-66. 
[9] Segal, I. E., A non-commutative extension of abstract integration, Ann. of Math. 57 (1953), 401-457.

[10] Umegaki, H., Conditional expectation in an operator algebra, II, Tôhoku Math. J. 8 (1956), 86-100.

[11] von Neumann, J., On rings of operators, Reduction theory, Ann. of Math. 50 (1949), 401-485. 